\title{
Tandem Amination/Oxetane Ring Opening towards Benzomorpholines
}

Lindsey G. DeRatt, Chao-Yuan Wang, Scott D. Kuduk

Janssen R\&D, 1400 McKean Road, Spring House, PA, 19477, United States

Table of Contents

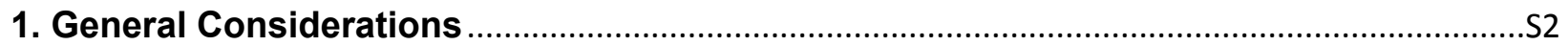

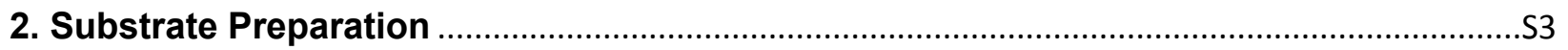

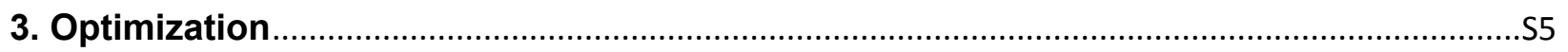

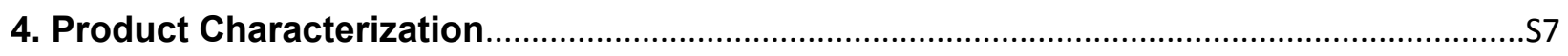

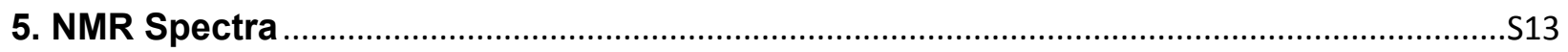




\section{General Considerations}

All reagents were purchased from commercial vendors and used without further purification. All oxetane reagents used were purchased from either Pharmablock or Combi-Blocks. DriSolv solvents were purchased from Sigma. Analytical thin layer chromatography (TLC) was performed using $250 \mu \mathrm{m}$ Silica Gel 60 F254 pre-coated plates. Flash column chromatography was performed using an Isco with RediSep silica columns. Proton nuclear magnetic resonance (1H NMR) spectra were recorded using a Bruker AVANCE III HD NanoBay 400 NMR spectrometer. Chemical shifts $(\delta)$ are reported in parts per million (ppm) downfield relative to tetramethylsilane (TMS, $0.0 \mathrm{ppm}), \mathrm{CDCl}_{3}(7.26 \mathrm{ppm}), \mathrm{MeOD}$ (3.31 ppm), or $\left(\mathrm{CD}_{3}\right)_{2} \mathrm{SO}(2.50 \mathrm{ppm})$. Coupling constants $(\mathrm{J})$ are reported in $\mathrm{Hz}$. Multiplicities are reported using the following abbreviations: $s$, singlet; $d$, doublet; $t$, triplet; $q$, quartet; $p$, pentet; $m$, multiplet; $b$, broad; Carbon-13 nuclear magnetic resonance (13C NMR) spectra were recorded using a Bruker AVANCE III HD NanoBay $400 \mathrm{NMR}$ spectrometer at $101 \mathrm{MHz}$. Chemical shifts are reported in ppm relative to the carbon resonance of $\mathrm{CDCl}_{3}$ (77.23 ppm), MeOD (49.01 ppm), or $\left(\mathrm{CD}_{3}\right)_{2} \mathrm{SO}$ (39.58 ppm). High resolution mass spectra (HRMS) are reported as $\mathrm{m} / \mathrm{z}$ (relative ratio). A Waters Xevo G2-XS QTof instrument was used for high resolution mass analysis. Accurate $\mathrm{m} / \mathrm{z}$ are reported for the molecular ion $[M+H]+$ or $[M-$ H]-. HRMS was unobtainable for 3-((3-bromonaphthalen-2-yl)oxy)oxetane, 3-(2-bromo-4-methoxy phenoxy)oxetane, 3-(2-bromo-4-nitrophenoxy)oxetane, 3-(2-bromo-6-chlorophenoxy)oxetane, 3-(2bromo-4-nitrophenoxy)-3-methyloxetane, methyl 4-bromo-3-(oxetan-3-yloxy)benzoate due to poor ionization. When heating is indicated in procedure, the reaction was performed using a IKA hot plate with an aluminum block and a thermocouple. A Biotage Initiator+ microwave reactor was used for the Cu-catalyzed reactions. 


\section{Substrate Preparation}<smiles>Brc1cc2ccccc2cc1OC1COC1</smiles>

3-((3-Bromonaphthalen-2-yl)oxy)oxetane (23). To a microwave vial was added 3-bromo-2naphthol $(3.0 \mathrm{~g}, 13.4 \mathrm{mmol}), 3$-iodooxetane $(3.5 \mathrm{~mL}, 2.14 \mathrm{~g} / \mathrm{mL}, 40.1 \mathrm{mmol}), \mathrm{Cs}_{2} \mathrm{CO}_{3}(13.1 \mathrm{~g}, 40.1$ $\mathrm{mmol}$ ) and $\mathrm{MeCN}(40 \mathrm{~mL})$. The mixture was heated at $75{ }^{\circ} \mathrm{C}$ for $16 \mathrm{~h}$ then filtered and concentrated. Purification by silica gel column chromatography (0-15\% EtOAc/heptane) afforded the title compound as a yellow solid $\left(2.97 \mathrm{~g}, 80 \%\right.$ yield). ${ }^{1} \mathrm{H}$ NMR $(400 \mathrm{MHz}$, Chloroform- $d) \delta 8.09(\mathrm{~s}, 1 \mathrm{H}), 7.73-7.62(\mathrm{~m}$, $2 \mathrm{H}), 7.49-7.42(\mathrm{~m}, 1 \mathrm{H}), 7.41-7.34(\mathrm{~m}, 1 \mathrm{H}), 6.68(\mathrm{~s}, 1 \mathrm{H}), 5.42-5.30(\mathrm{~m}, 1 \mathrm{H}), 5.12-5.03(\mathrm{~m}, 2 \mathrm{H})$, $4.93-4.85(\mathrm{~m}, 2 \mathrm{H}) .{ }^{13} \mathrm{C}\left\{{ }^{1} \mathrm{H}\right\}$ NMR $(101 \mathrm{MHz}$, Chloroform-d) $\delta 150.7,133.2,132.9,129.8,127.0$, $126.8,126.6,125.0,113.2,107.5,77.8,71.2$.<smiles>COc1ccc(OC2COC2)c(Br)c1</smiles>

3-(2-Bromo-4-methoxyphenoxy)oxetane. To a microwave vial was added 2-bromo-4methoxyphenol $(750 \mathrm{mg}, 3.6 \mathrm{mmol}), \mathrm{Cs}_{2} \mathrm{CO}_{3}(3.5 \mathrm{~g}, 10.9 \mathrm{mmol})$ and $\mathrm{MeCN}(5.0 \mathrm{~mL})$. 3-Iodooxetane $(0.93 \mathrm{~mL}, 2.14 \mathrm{~g} / \mathrm{mL}, 10.9 \mathrm{mmol})$ was added and the mixture was heated at $80^{\circ} \mathrm{C}$ for $16 \mathrm{~h}$. The mixture was then filtered and concentrated. Purification by silica gel column chromatography (10-50\% EtOAc/heptane) afforded the title compound as a colorless oil $\left(884 \mathrm{mg}, 94 \%\right.$ yield). ${ }^{1} \mathrm{H}$ NMR $(400 \mathrm{MHz}$, Chloroform-d) $\delta 7.14(\mathrm{~d}, J=3.0 \mathrm{~Hz}, 1 \mathrm{H}), 6.76(\mathrm{dd}, J=8.9,3.0 \mathrm{~Hz}, 1 \mathrm{H}), 6.45(\mathrm{~d}, J=8.9 \mathrm{~Hz}, 1 \mathrm{H}), 5.19$ - $5.13(\mathrm{~m}, 1 \mathrm{H}), 4.97-4.91(\mathrm{~m}, 2 \mathrm{H}), 4.83(\mathrm{dd}, \mathrm{J}=7.3,5.7 \mathrm{~Hz}, 2 \mathrm{H}), 3.76(\mathrm{~s}, 3 \mathrm{H}) .{ }^{13} \mathrm{C}\left\{{ }^{1} \mathrm{H}\right\} \mathrm{NMR}(101$ $\mathrm{MHz}$, Chloroform-d) $\delta 154.7,147.6,119.3,114.4,113.7,112.7,78.0,71.9,55.9$.<smiles>FC(F)(F)c1ccc(Br)c(OC2COC2)c1</smiles>

3-(2-Bromo-5-(trifluoromethyl)phenoxy)oxetane. To a microwave vial was added 2-bromo-5trifluoromethylphenol $(630 \mathrm{mg}, 2.6 \mathrm{mmol}), \mathrm{Cs}_{2} \mathrm{CO}_{3}(2.6 \mathrm{~g}, 7.9 \mathrm{mmol})$ and $\mathrm{MeCN}(5.0 \mathrm{~mL})$. 3Iodooxetane $(0.68 \mathrm{ml}, 2.14 \mathrm{~g} / \mathrm{ml}, 7.9 \mathrm{mmol})$ was added and the mixture was heated at $80^{\circ} \mathrm{C}$ for $24 \mathrm{~h}$. The mixture was then filtered and concentrated. Purification by silica gel column chromatography (10$50 \%$ EtOAc/heptane) afforded the title compound as a white solid (612 mg, 79\% yield). Spectroscopic data matched that previously reported. ${ }^{1}$<smiles>O=[N+]([O-])c1ccc(OC2COC2)c(Br)c1</smiles>

3-(2-Bromo-4-nitrophenoxy)oxetane. To a solution of oxetan-3-ol (577 $\mu \mathrm{L}, 1.17 \mathrm{~g} / \mathrm{mL}, 9.1 \mathrm{mmol})$ in DMF $(10 \mathrm{~mL})$ at $0^{\circ} \mathrm{C}$ was added $\mathrm{NaH}(0.36 \mathrm{~g}, 9.1 \mathrm{mmol})$. The mixture was stirred for $0.5 \mathrm{~h}$ then 3bromo-4-fluoronitrobenzene $(1 \mathrm{~g}, 4.6 \mathrm{mmol})$ was added. The mixture was warmed to room temperature and stirred for $0.5 \mathrm{~h}$ then diluted with EtOAc and quenched with water. Transferred to a separatory funnel and extracted with EtOAc. The combined organic layers were washed with brine, dried over sodium sulfate, filtered and concentrated. Purification by column chromatography (10-50\% EtOAc/heptane) afforded the title compound as a pale yellow solid $\left(1.2 \mathrm{~g}, 93 \%\right.$ yield). ${ }^{1} \mathrm{H}$ NMR (400 $\mathrm{MHz}$, Chloroform- $d) \delta 8.50(\mathrm{~d}, J=2.7 \mathrm{~Hz}, 1 \mathrm{H}), 8.16(\mathrm{dd}, J=9.0,2.7 \mathrm{~Hz}, 1 \mathrm{H}), 6.50(\mathrm{~d}, J=9.0 \mathrm{~Hz}, 1 \mathrm{H})$, $5.40-5.31(\mathrm{~m}, 1 \mathrm{H}), 5.09-5.01(\mathrm{~m}, 2 \mathrm{H}), 4.90-4.79(\mathrm{~m}, 2 \mathrm{H}) .{ }^{13} \mathrm{C}\left\{{ }^{1} \mathrm{H}\right\} \mathrm{NMR}(101 \mathrm{MHz}$, Chloroformd) $\delta 158.2,142.1,129.7,124.6,112.3,111.5,77.1,72.0$. 
<smiles>Clc1cccc(Br)c1OC1COC1</smiles>

3-(2-Bromo-6-chlorophenoxy)oxetane. To a microwave vial was added 2-bromo-6-chlorophenol $(1.0 \mathrm{~g}, 4.8 \mathrm{mmol}), \mathrm{Cs}_{2} \mathrm{CO}_{3}(4.5 \mathrm{~g}, 13.7 \mathrm{mmol})$ and $\mathrm{MeCN}(9 \mathrm{~mL}) .3$-Iodooxetane $(0.83 \mathrm{~mL}, 2.14 \mathrm{~g} / \mathrm{mL}$, $9.6 \mathrm{mmol}$ ) was added and the mixture stirred at $80{ }^{\circ} \mathrm{C}$ for $48 \mathrm{~h}$. The mixture was then filtered and concentrated. Purification by silica gel column chromatography (0-20\% EtOAc/heptane) provided the title compound as a yellow oil $\left(1.2 \mathrm{~g}, 97 \%\right.$ yield). ${ }^{1} \mathrm{H}$ NMR $(400 \mathrm{MHz}$, Chloroform- $d) \delta 7.46$ (dd, $J=8.1$, $1.5 \mathrm{~Hz}, 1 \mathrm{H}), 7.34(\mathrm{dd}, J=8.1,1.5 \mathrm{~Hz}, 1 \mathrm{H}), 6.94(\mathrm{t}, J=8.1 \mathrm{~Hz}, 1 \mathrm{H}), 5.14-4.98(\mathrm{~m}, 3 \mathrm{H}), 4.93-4.84$ $(\mathrm{m}, 2 \mathrm{H}) .{ }^{13} \mathrm{C}\left\{{ }^{1} \mathrm{H}\right\} \mathrm{NMR}(101 \mathrm{MHz}$, Chloroform-d $) \delta 152.2,131.9,129.7,128.7,125.9,118.3,79.2,77.0$.<smiles>Clc1ncccc1OC1COC1</smiles>

2-Chloro-3-(oxetan-3-yloxy)pyridine. To a microwave vial was added 2-chloro-3-hydroxypyridine $(950 \mathrm{mg}, 7.3 \mathrm{mmol})$ and $\mathrm{Cs}_{2} \mathrm{CO}_{3}(4.8 \mathrm{~g}, 14.7 \mathrm{mmol})$. MeCN $(10 \mathrm{~mL})$ followed by 3-iodooxetane $(0.95$ $\mathrm{mL}, 2.14 \mathrm{~g} / \mathrm{mL}, 11 \mathrm{mmol}$ ) were added. The mixture was heated at $80{ }^{\circ} \mathrm{C}$ for $24 \mathrm{~h}$ then filtered and concentrated. Purification by silica gel column chromatography $(0-50 \%$ EtOAc/heptane) afforded the title compound as a colorless oil ( $820 \mathrm{mg}, 60 \%$ yield). ${ }^{1} \mathrm{H}$ NMR $(400 \mathrm{MHz}$, Chloroform-d) $\delta 8.05$ (dd, $J$ $=4.7,1.3 \mathrm{~Hz}, 1 \mathrm{H}), 7.17(\mathrm{dd}, J=8.1,4.7 \mathrm{~Hz}, 1 \mathrm{H}), 6.79(\mathrm{dd}, J=8.1,1.3 \mathrm{~Hz}, 1 \mathrm{H}), 5.30-5.21(\mathrm{~m}, 1 \mathrm{H})$, $5.04-4.95(\mathrm{~m}, 2 \mathrm{H}), 4.85(\mathrm{dd}, J=7.6,5.3 \mathrm{~Hz}, 2 \mathrm{H}) .{ }^{13} \mathrm{C}\left\{{ }^{1} \mathrm{H}\right\}$ NMR $(101 \mathrm{MHz}$, Chloroform-d) $\delta 149.0$, $141.5,141.2,123.0,120.1,77.4,71.4$. HRMS (ESI-TOF) $\mathrm{m} / \mathrm{z}$ : $[\mathrm{M}+\mathrm{H}]+$ Calcd for $\mathrm{C}_{8} \mathrm{H}_{9} \mathrm{ClNO}_{2} 186.0322$; Found 186.0331.<smiles>CC1(Oc2ccc([N+](=O)[O-])cc2Br)COC1</smiles>

3-(2-Bromo-4-nitrophenoxy)-3-methyloxetane. To a vial containing 3-bromo-4fluoronitrobenzene $(330 \mathrm{mg}, 1.5 \mathrm{mmol})$ and $\mathrm{Cs}_{2} \mathrm{CO}_{3}(1.4 \mathrm{~g}, 4.3 \mathrm{mmol})$ was added 3-methyloxetan-3-ol (139 $\mathrm{mg}, 1.58 \mathrm{mmol}$ ) and DMF $(6 \mathrm{~mL})$. The mixture was stirred at room temperature overnight then diluted with EtOAc and water. The organics were extracted with EtOAc three times then washed with brine, dried over sodium sulfate, filtered and concentrated. Purification by column chromatography ( 0 $20 \%$ EtOAc/heptane) afforded the title compound as a white solid (408 mg, 94\% yield). ${ }^{1} \mathrm{H}$ NMR (400 $\mathrm{MHz}$, Chloroform- $d$ ) $\delta 8.50(\mathrm{~d}, J=2.7 \mathrm{~Hz}, 1 \mathrm{H}), 8.13(\mathrm{dd}, J=9.1,2.7 \mathrm{~Hz}, 1 \mathrm{H}), 6.39(\mathrm{~d}, J=9.1 \mathrm{~Hz}, 1 \mathrm{H})$, $4.99(\mathrm{~d}, J=6.8 \mathrm{~Hz}, 2 \mathrm{H}), 4.67(\mathrm{~d}, J=7.6 \mathrm{~Hz}, 2 \mathrm{H}), 1.83(\mathrm{~s}, 3 \mathrm{H}) .{ }^{13} \mathrm{C}\left\{{ }^{1} \mathrm{H}\right\} \mathrm{NMR}(101 \mathrm{MHz}$, Chloroformd) $\delta 156.9,141.7,129.9,124.2,113.33,113.2,81.6,79.4,21.0$.<smiles>COC(=O)c1ccc(Br)c(OC2COC2)c1</smiles>

Methyl 4-bromo-3-(oxetan-3-yloxy)benzoate. To a microwave vial was added methyl 4-bromo-3hydroxybenzoate $(460 \mathrm{mg}, 1.9 \mathrm{mmol}), \mathrm{Cs}_{2} \mathrm{CO}_{3}(1.8 \mathrm{~g}, 5.5 \mathrm{mmol})$ and $\mathrm{MeCN}(3.8 \mathrm{~mL})$. 3-Iodooxetane $(0.33 \mathrm{~mL}, 2.14 \mathrm{~g} / \mathrm{mL}, 3.9 \mathrm{mmol})$ was added and the mixture stirred at $80^{\circ} \mathrm{C}$ for $48 \mathrm{~h}$. The mixture was filtered and concentrated. Purification by silica gel column chromatography (0-60\% EtOAc/heptane) provided the title compound as a white solid ( $460 \mathrm{mg}, 82 \%$ yield). ${ }^{1} \mathrm{H}$ NMR ( $400 \mathrm{MHz}$, Chloroform- $d$ ) $\delta$ $7.65(\mathrm{~d}, J=8.2 \mathrm{~Hz}, 1 \mathrm{H}), 7.54(\mathrm{dd}, J=8.2,1.8 \mathrm{~Hz}, 1 \mathrm{H}), 7.10(\mathrm{~d}, J=1.8 \mathrm{~Hz}, 1 \mathrm{H}), 5.36-5.27(\mathrm{~m}, 1 \mathrm{H})$, $5.07-5.00(\mathrm{~m}, 2 \mathrm{H}), 4.84(\mathrm{dd}, J=8.0,5.2 \mathrm{~Hz}, 2 \mathrm{H}), 3.91(\mathrm{~s}, 3 \mathrm{H}) .{ }^{13} \mathrm{C}\left\{{ }^{1} \mathrm{H}\right\} \mathrm{NMR}(101 \mathrm{MHz}, \mathrm{Chloroform}-d)$ $\delta 166.0,153.2,133.9,130.6,123.7,117.9,113.2,77.6,71.3,52.5$. 


\section{Optimization}

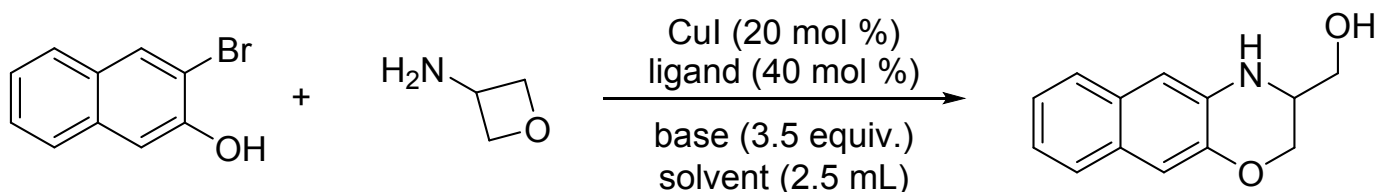

(1.2 - 3 equiv.)

MW or $\Delta$

Cu General Optimization Procedure: To a $5 \mathrm{~mL}$ microwave vial was added CuI ( $38 \mathrm{mg}, 0.2 \mathrm{mmol}$ ), ligand $(0.4 \mathrm{mmol}), 3$-bromo-2-naphthol $(223 \mathrm{mg}, 1.0 \mathrm{mmol})$, and base $(3.5 \mathrm{mmol})$. Solvent $(2.5 \mathrm{~mL})$ followed by 3 -oxetanamine ( $140 \mathrm{uL}, 1.04 \mathrm{~g} / \mathrm{mL}, 2.0 \mathrm{mmol}$ ) were added. The vial was capped and sealed, and the mixture heated in the microwave at $120^{\circ} \mathrm{C}$ for $4 \mathrm{~h}$ unless otherwise indicated in Table 1 . The mixture was diluted with EtOAc, filtered over a silica plug and concentrated. Purification by silica gel column chromatography (EtOAc/heptane) provided the product.

\begin{tabular}{|c|c|c|c|c|}
\hline $\begin{array}{l}\text { catalyst } \\
(20 \%)\end{array}$ & $\begin{array}{l}\text { ligand } \\
(40 \%)\end{array}$ & $\begin{array}{c}\text { base } \\
\text { ( } 3.5 \text { equiv) }\end{array}$ & solvent & $\begin{array}{l}\text { isolated } \\
\text { yield }(\%)\end{array}$ \\
\hline CuI & L1 & $\mathrm{K}_{2} \mathrm{CO}_{3}$ & DMF & 47 \\
\hline CuI & L2 & $\mathrm{K}_{2} \mathrm{CO}_{3}$ & DMF & $<10$ \\
\hline CuI & L3 & $\mathrm{K}_{2} \mathrm{CO}_{3}$ & DMF & 24 \\
\hline CuI & L4 & $\mathrm{K}_{2} \mathrm{CO}_{3}$ & DMF & 45 \\
\hline CuI & L5 & $\mathrm{K}_{2} \mathrm{CO}_{3}$ & DMF & $74(72 *)$ \\
\hline CuI & L6 & $\mathrm{K}_{2} \mathrm{CO}_{3}$ & DMF & 74 \\
\hline CuI & L7 & $\mathrm{K}_{2} \mathrm{CO}_{3}$ & DMF & $<10$ \\
\hline CuI & None & $\mathrm{K}_{2} \mathrm{CO}_{3}$ & DMF & 39 \\
\hline CuI & L5 & $\mathrm{Cs}_{2} \mathrm{CO}_{3}$ & DMF & 70 \\
\hline CuI & L5 & $\mathrm{Na}_{2} \mathrm{CO}_{3}$ & DMF & 27 \\
\hline CuI & L5 & $\mathrm{NaOtBu}$ & DMF & 59 \\
\hline CUI & L5 & $\mathrm{K}_{3} \mathrm{PO}_{4}$ & DMF & 32 \\
\hline CuI & L5 & none & DMF & 0 \\
\hline CuI & L5 & $\mathrm{K}_{2} \mathrm{CO}_{3}$ & 1,4-dioxane & $55^{*}$ \\
\hline CuI & L5 & $\mathrm{K}_{2} \mathrm{CO}_{3}$ & toluene & $<10^{*}$ \\
\hline CuI $(10 \%)$ & L5 (20\%) & $\mathrm{K}_{2} \mathrm{CO}_{3}$ & DMF & $84 / 86 \%$ (2 runs) \\
\hline CuI (5\%) & L5 (10\%) & $\mathrm{K}_{2} \mathrm{CO}_{3}$ & DMF & 77 \\
\hline
\end{tabular}

*Heating in aluminum heating block with thermocouple for $16 \mathrm{~h}$

(1)




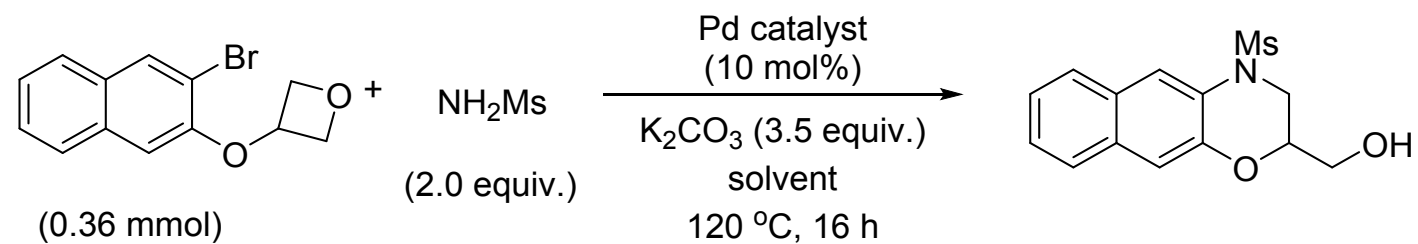

Pd General Optimization Procedure: To a $5 \mathrm{~mL}$ microwave vial was added 3-((3-bromonaphthalen2-yl)oxy)oxetane (100 mg, $0.36 \mathrm{mmol})$, methanesulfonamide $(70 \mathrm{mg}, 0.72 \mathrm{mmol})$, Pd catalyst $(0.036$ $\mathrm{mmol})$, and $\mathrm{K}_{2} \mathrm{CO}_{3}(173 \mathrm{mg}, 1.25 \mathrm{mmol})$. The vial was capped then evacuated and backfilled with Argon three times. 1,4 -Dioxane $(1.0 \mathrm{~mL})$ was added and the vial placed in a heating block at $120{ }^{\circ} \mathrm{C}$. The reaction mixture was stirred overnight $(16 \mathrm{~h})$ then diluted with EtOAc and filtered over a pad of silica and concentrated. Purification by silica gel column chromatography (EtOAc/heptane) provided the product.

\begin{tabular}{|c|c|c|c|}
\hline catalyst & base & solvent & isolated yield (\%) \\
\hline t-BuBrettPhos Pd G3 & $\mathrm{K}_{2} \mathrm{CO}_{3}$ & DMF & 55 \\
\hline tBuXPhos Pd G3 & $\mathrm{K}_{2} \mathrm{CO}_{3}$ & DMF & 70 \\
\hline XantPhos Pd G3 & $\mathrm{K}_{2} \mathrm{CO}_{3}$ & DMF & $<10$ \\
\hline BrettPhos Pd G3 & $\mathrm{K}_{2} \mathrm{CO}_{3}$ & DMF & 84 \\
\hline RuPhos Pd G3 & $\mathrm{K}_{2} \mathrm{CO}_{3}$ & DMF & 60 \\
\hline CyJohnPhos Pd G3 & $\mathrm{K}_{2} \mathrm{CO}_{3}$ & DMF & 0 \\
\hline SPhos Pd G3 & $\mathrm{K}_{2} \mathrm{CO}_{3}$ & DMF & 23 \\
\hline XPhos Pd G3 & $\mathrm{K}_{2} \mathrm{CO}_{3}$ & DMF & 75 \\
\hline $\mathrm{P}(t \mathrm{Bu})_{3} \mathrm{Pd} \mathrm{G} 4$ & $\mathrm{~K}_{2} \mathrm{CO}_{3}$ & DMF & 0 \\
\hline BrettPhos Pd G4 & $\mathrm{K}_{2} \mathrm{CO}_{3}$ & DMF & 85 \\
\hline BrettPhos Pd G3 & $\mathrm{Cs}_{2} \mathrm{CO}_{3}$ & DMF & 29 \\
\hline BrettPhos Pd G3 (10 \%) & $\mathrm{K}_{2} \mathrm{CO}_{3}$ & 1,4-dioxane & 89 \\
\hline BrettPhos Pd G3 (5\%) & $\mathrm{K}_{2} \mathrm{CO}_{3}$ & 1,4-dioxane & 99 \\
\hline BrettPhos Pd G3 (2.5\%) & $\mathrm{K}_{2} \mathrm{CO}_{3}$ & 1,4-dioxane & 97 \\
\hline BrettPhos Pd G3 (1\%) & $\mathrm{K}_{2} \mathrm{CO}_{3}$ & 1,4-dioxane & 92 \\
\hline
\end{tabular}




\section{Product Characterization}

Compounds prepared according to general procedures. Any deviation for specific examples is indicated.

General Procedure 1: To a 2.0 - $5.0 \mathrm{~mL}$ Biotage microwave vial was added CuI (19 mg, 0.1 mmol), [(2,6-dimethylphenyl)carbamoyl]formic acid (39 mg, $0.2 \mathrm{mmol}$ ), aryl halide (1.0 $\mathrm{mmol})$, and $\mathrm{K}_{2} \mathrm{CO}_{3}(485 \mathrm{mg}, 3.5 \mathrm{mmol})$. DMF $(2.5 \mathrm{~mL})$ followed by oxetanamine $(2.0 \mathrm{mmol})$ were then added. The vial was capped then placed in the microwave reactor at $120^{\circ} \mathrm{C}$ for 4 h. The mixture was diluted with EtOAc, filtered over a silica plug and concentrated. Purification by silica gel column chromatography (EtOAc/heptane) provided the product.<smiles>OCC1COc2cc3ccccc3cc2N1</smiles>

(3,4-Dihydro-2H-naphtho[2,3-b][1,4]oxazin-3-yl)methanol (12). Prepared following General Procedure 1 using 3-bromo-2-naphthol (Run 1: $234 \mathrm{mg}$; Run 2: $234 \mathrm{mg}$ ) and 3-oxetanamine (140 $\mu \mathrm{L})$. Purification by column chromatography (50-80\% EtOAc/heptane) afforded the title compound as a pale yellow solid (Run 1: $194 \mathrm{mg}, 86 \%$ yield, Run 2: $189 \mathrm{mg}, 84 \%$ yield). ${ }^{1} \mathrm{H}$ NMR ( $400 \mathrm{MHz}$, Chloroform- $d$ ) $\delta 7.59(\mathrm{~d}, J=8.0 \mathrm{~Hz}, 1 \mathrm{H}), 7.54(\mathrm{~d}, J=8.0 \mathrm{~Hz}, 1 \mathrm{H}), 7.25-7.15(\mathrm{~m}, 3 \mathrm{H}), 6.95(\mathrm{~s}, 1 \mathrm{H}), 4.48(\mathrm{br} \mathrm{s}, 1 \mathrm{H})$, $4.30(\mathrm{~d}, J=10.6 \mathrm{~Hz}, 1 \mathrm{H}), 4.16(\mathrm{dd}, J=10.8,5.6 \mathrm{~Hz}, 1 \mathrm{H}), 3.85-3.78(\mathrm{~m}, 1 \mathrm{H}), 3.76-3.63(\mathrm{~m}, 1 \mathrm{H})$, $1.78(\mathrm{t}, J=4.9 \mathrm{~Hz}, 1 \mathrm{H}) .{ }^{13} \mathrm{C}\left\{{ }^{1} \mathrm{H}\right\} \mathrm{NMR}(101 \mathrm{MHz}$, Chloroform-d) $\delta 144.70,133.7,130.2,128.3,126.5$, $125.3,124.2,122.9,111.9,109.3,66.1,62.9,51.0$. HRMS (ESI-TOF) m/z: $[M+H]+$ Calcd for $\mathrm{C}_{13} \mathrm{H}_{14} \mathrm{NO}_{2}$ 216.1025; Found 216.1031.<smiles>N#Cc1ccc2c(c1)NC(CO)CO2</smiles>

3-(Hydroxymethyl)-3,4-dihydro-2H-benzo[b][1,4]oxazine-6-carbonitrile (13). Prepared following General Procedure 1 using 3-bromo-4-hydroxybenzonitrile (Run 1: $203 \mathrm{mg}$; Run 2: $200 \mathrm{mg}$ ) and 3-oxetanamine $(140 \mu \mathrm{L})$. Purification by column chromatography (20-60\% EtOAc/heptane) afforded the title compound as a light tan oil (Run 1: $133 \mathrm{mg}, 68 \%$ yield; Run 2: $132 \mathrm{mg} ; 69 \%$ yield). ${ }^{1} \mathrm{H}$ NMR $(400 \mathrm{MHz}$, Chloroform- $d$ ) $\delta 6.95$ (dd, $J=8.3,1.9 \mathrm{~Hz}, 1 \mathrm{H}), 6.87(\mathrm{~d}, J=1.9 \mathrm{~Hz}, 1 \mathrm{H}), 6.81(\mathrm{~d}, J=8.3$ $\mathrm{Hz}, 1 \mathrm{H}), 4.41(\mathrm{~s}, 1 \mathrm{H}), 4.25(\mathrm{dd}, J=10.9,2.8 \mathrm{~Hz}, 1 \mathrm{H}), 4.10(\mathrm{dd}, J=10.9,5.7 \mathrm{~Hz}, 1 \mathrm{H}), 3.76(\mathrm{dd}, J=$ 10.4, 4.7 Hz, $1 \mathrm{H}), 3.69-3.55(\mathrm{~m}, 2 \mathrm{H}), 2.10(\mathrm{br} \mathrm{s}, 1 \mathrm{H}) .{ }^{13} \mathrm{C}\left\{{ }^{1} \mathrm{H}\right\} \mathrm{NMR}(101 \mathrm{MHz}$, Chloroform-d) $\delta 147.4$, 133.6, 123.2, 119.6, 118.4, 117.3, 104.4, 66.1, 62.4, 50.6. HRMS (ESI-TOF) m/z: $[\mathrm{M}+\mathrm{H}]^{+}$Calcd for $\mathrm{C}_{10} \mathrm{H}_{11} \mathrm{~N}_{2} \mathrm{O}_{2}$ 191.0821; Found 191.0826.<smiles>OCC1COc2cc(C(F)(F)F)ccc2N1</smiles>

(7-(Trifluoromethyl)-3,4-dihydro-2H-benzo[b][1,4]oxazin-3-yl)methanol (14). Prepared following General Procedure 1 using 2-bromo-5-trifluoromethylphenol (Run 1: $255 \mathrm{mg}$; Run 2: $244 \mathrm{mg}$ ) and 3-oxetanamine $(140 \mu \mathrm{L})$. Purification by column chromatography (30-100\% EtOAc/heptane) afforded the title compound as a tan oil (Run 1: $152 \mathrm{mg}, 62 \%$ yield; Run $2: 132 \mathrm{mg}, 56 \%$ yield). ${ }^{1} \mathrm{H}$ NMR $(400 \mathrm{MHz}$, Chloroform-d) $\delta 7.07-7.00(\mathrm{~m}, 2 \mathrm{H}), 6.64(\mathrm{~d}, \mathrm{~J}=8.5 \mathrm{~Hz}, 1 \mathrm{H}), 4.41(\mathrm{br} \mathrm{s}, 1 \mathrm{H}), 4.20$ (dd, $J=10.9,2.8 \mathrm{~Hz}, 1 \mathrm{H}), 4.09(\mathrm{dd}, J=10.9,5.2 \mathrm{~Hz}, 1 \mathrm{H}), 3.77(\mathrm{dd}, J=10.1,4.6 \mathrm{~Hz}, 1 \mathrm{H}), 3.70-3.56$ $(\mathrm{m}, 2 \mathrm{H}), 1.75(\mathrm{~s}, 1 \mathrm{H}) .{ }^{13} \mathrm{C}\left\{{ }^{1} \mathrm{H}\right\} \mathrm{NMR}(101 \mathrm{MHz}$, Chloroform-d $) \delta 142.9,135.9,124.5(\mathrm{q}, J=270.7 \mathrm{~Hz})$, $120.3(q, J=32.9 \mathrm{~Hz}), 119.0(\mathrm{q}, J=4.0 \mathrm{~Hz}), 114.7,113.9(\mathrm{q}, J=3.8 \mathrm{~Hz}), 65.5,62.7,50.9 .{ }^{19} \mathrm{~F}\left\{{ }^{1} \mathrm{H}\right\}$ NMR (376 MHz, Chloroform-d) $\delta$-61.3. HRMS (ESI-TOF) m/z: $[\mathrm{M}+\mathrm{H}]^{+}$Calcd for $\mathrm{C}_{10} \mathrm{H}_{11} \mathrm{~F}_{3} \mathrm{NO}_{2} 234.0742$; Found 234.0750. 
<smiles>COc1ccc2c(c1)NC(CO)CO2</smiles>

(6-Methoxy-3,4-dihydro-2H-benzo[b][1,4]oxazin-3-yl)methanol (15). Prepared following General Procedure 1 using 2-bromo-4-methoxyphenol (Run 1: $224 \mathrm{mg}$; Run 2: $215 \mathrm{mg}$ ) and 3oxetanamine $(140 \mu \mathrm{L})$. Purification by column chromatography (20-60\% EtOAc/heptane) afforded the title compound as a brown oil (Run 1: $120 \mathrm{mg}, 57 \%$ yield; Run 2: $106 \mathrm{mg}, 52 \%$ yield). ${ }^{1} \mathrm{H}$ NMR (400 $\mathrm{MHz}$, Chloroform-d) $\delta 6.80-6.64(\mathrm{~m}, 1 \mathrm{H}), 6.30-6.13(\mathrm{~m}, 2 \mathrm{H}), 4.15(\mathrm{dd}, \mathrm{J}=10.8,2.9,1 \mathrm{H}), 4.10(\mathrm{br}$ $\mathrm{s}, 1 \mathrm{H}), 4.05(\mathrm{dd}, \mathrm{J}=10.9,5.6,1 \mathrm{H}), 3.79-3.71(\mathrm{~m}, 4 \mathrm{H}), 3.71-3.63(\mathrm{~m}, 1 \mathrm{H}), 3.61-3.54(\mathrm{~m}, 1 \mathrm{H})$, $1.87-1.76(\mathrm{~m}, 1 \mathrm{H}) .{ }^{13} \mathrm{C}\left\{{ }^{1} \mathrm{H}\right\}$ NMR $(101 \mathrm{MHz}$, Chloroform-d) $\delta 154.6,137.9,133.2,116.9,103.8$, 101.6, 65.7, 62.9, 55.6, 51.2. HRMS (ESI-TOF) $\mathrm{m} / \mathrm{z}:[\mathrm{M}+\mathrm{H}]^{+}$Calcd for $\mathrm{C}_{10} \mathrm{H}_{14} \mathrm{NO}_{3}$ 196.0974; Found 196.0980 .<smiles>COc1ccc2c(c1)OCC(CO)N2</smiles>

(7-Methoxy-3,4-dihydro-2H-benzo[b][1,4]oxazin-3-yl)methanol (16). Prepared following General Procedure 1 using 2-bromo-5-methoxyphenol (Run 1: $206 \mathrm{mg}$; Run 2: $215 \mathrm{mg}$ ) and 3oxetanamine $(140 \mu \mathrm{L})$. Purification by column chromatography (30-70\% EtOAc/heptane) afforded the title compound as a dark brown oil (Run 1: $86 \mathrm{mg}, 44 \%$ yield; Run $2: 77 \mathrm{mg}, 37 \%$ yield). ${ }^{1} \mathrm{H}$ NMR (400 $\mathrm{MHz}$, Chloroform- $d) \delta 6.60(\mathrm{~d}, J=8.3 \mathrm{~Hz}, 1 \mathrm{H}), 6.44-6.38(\mathrm{~m}, 2 \mathrm{H}), 4.20(\mathrm{dd}, \mathrm{J}=10.8,2.8 \mathrm{~Hz}, 1 \mathrm{H})$, $4.06(\mathrm{dd}, J=10.8,6.2 \mathrm{~Hz}, 1 \mathrm{H}), 3.76-3.69(\mathrm{~m}, 4 \mathrm{H}), 3.65(\mathrm{dd}, \mathrm{J}=10.8,6.8 \mathrm{~Hz}, 1 \mathrm{H}), 3.55-3.48(\mathrm{~m}$, $1 \mathrm{H}) .{ }^{13} \mathrm{C}\left\{{ }^{1} \mathrm{H}\right\}$ NMR $(101 \mathrm{MHz}$, Chloroform-d) $\delta 153.5,144.7,125.9,117.1,107.5,102.8,66.3,62.5$, 55.7, 51.2. HRMS (ESI-TOF) $\mathrm{m} / \mathrm{z}:[\mathrm{M}+\mathrm{H}]^{+}$Calcd for $\mathrm{C}_{10} \mathrm{H}_{14} \mathrm{NO}_{3}$ 196.0974; Found 196.0979.<smiles>OCC1COc2c(Cl)cccc2N1</smiles>

(8-Chloro-3,4-dihydro-2H-benzo[b][1,4] oxazin-3-yl)methanol (17). Prepared following General Procedure 1 using 2-bromo-6-chlorophenol (Run 1: $207 \mathrm{mg}$; Run 2: $211 \mathrm{mg}$ ) and 3-oxetanamine (140 $\mu \mathrm{L})$. Purification by column chromatography (20-60\% EtOAc/heptane) afforded the title compound as a brown oil (Run 1: $133 \mathrm{mg}, 66 \%$ yield; Run 2: $134 \mathrm{mg}, 66 \%$ yield). ${ }^{1} \mathrm{H}$ NMR ( $400 \mathrm{MHz}$, Chloroform-d) $\delta$ $6.76-6.67(\mathrm{~m}, 2 \mathrm{H}), 6.53(\mathrm{dd}, \mathrm{J}=7.7,1.7 \mathrm{~Hz}, 1 \mathrm{H}), 4.28(\mathrm{dd}, J=10.8,2.8 \mathrm{~Hz}, 1 \mathrm{H}), 4.25-4.13(\mathrm{~m}$, $2 \mathrm{H}), 3.76(\mathrm{dd}, J=10.5,4.8 \mathrm{~Hz}, 1 \mathrm{H}), 3.70-3.55(\mathrm{~m}, 2 \mathrm{H}), 1.85(\mathrm{br} \mathrm{s}, 1 \mathrm{H}) .{ }^{13} \mathrm{C}\left\{{ }^{1} \mathrm{H}\right\} \mathrm{NMR}(101 \mathrm{MHz}$, Chloroform-d) $\delta$ 139.5, 134.0, 121.6, 121.5, 119.4, 114.1, 66.3, 62.5, 50.9. HRMS (ESI-TOF) m/z: $[\mathrm{M}+\mathrm{H}]+$ Calcd for $\mathrm{C}_{9} \mathrm{H}_{11} \mathrm{ClNO}_{2}$ 200.0478; Found 200.0484, 202.0457.<smiles>OCC1COc2cccc(F)c2N1</smiles>

(5-Fluoro-3,4-dihydro-2H-benzo[b][1,4]oxazin-3-yl)methanol (18). Prepared following General Procedure 1 using 2-bromo-3-fluorophenol (Run 1: $207 \mathrm{mg}$, Run 2: $201 \mathrm{mg}$ ) and 3-oxetanamine (140 $\mu \mathrm{L})$. Purification by column chromatography (20-50\% EtOAc/heptane) afforded the title compound as a brown oil (Run 1: $114 \mathrm{mg}, 57 \%$ yield; Run 2: $105 \mathrm{mg}, 55 \%$ yield). ${ }^{1} \mathrm{H}$ NMR (400 MHz, Chloroform-d) $\delta$ $6.67-6.54(\mathrm{~m}, 3 \mathrm{H}), 4.23(\mathrm{dd}, J=10.9,2.9 \mathrm{~Hz}, 1 \mathrm{H}), 4.12(\mathrm{dd}, J=10.9,5.8 \mathrm{~Hz}, 1 \mathrm{H}), 3.77(\mathrm{dd}, J=$ $10.7,5.1 \mathrm{~Hz}, 1 \mathrm{H}), 3.69(\mathrm{dd}, J=10.7,6.8 \mathrm{~Hz}, 1 \mathrm{H}), 3.63-3.56(\mathrm{~m}, 1 \mathrm{H}) .{ }^{13} \mathrm{C}\left\{{ }^{1} \mathrm{H}\right\} \mathrm{NMR}(101 \mathrm{MHz}$, Chloroform- $d) \delta 151.8(\mathrm{~d}, J=238.1 \mathrm{~Hz}), 145.0(\mathrm{~d}, J=5.8 \mathrm{~Hz}), 121.8(\mathrm{~d}, J=15.9 \mathrm{~Hz}), 117.2(\mathrm{~d}, J=$ 
$9.0 \mathrm{~Hz}), 112.1(\mathrm{~d}, J=2.6 \mathrm{~Hz}), 107.8(\mathrm{~d}, J=18.4 \mathrm{~Hz}), 65.9,62.5,50.5 .{ }^{19} \mathrm{~F}\left\{{ }^{1} \mathrm{H}\right\} \mathrm{NMR}(377 \mathrm{MHz}$, Chloroform-d) $\delta-136.1$. HRMS (ESI-TOF) m/z: [M+H] ${ }^{+}$Calcd for $\mathrm{C}_{9} \mathrm{H}_{11} \mathrm{FNO}_{2}$ 184.0774; Found 184.0780.<smiles>Cc1cccc2c1NC(CO)CO2</smiles>

(5-Methyl-3,4-dihydro-2H-benzo[b][1,4]oxazin-3-yl)methanol (19). Prepared following General Procedure 1 using 2-bromo-3-methylphenol (Run 1: $187 \mathrm{mg}$; Run 2: $194 \mathrm{mg}$ ) and 3oxetanamine $(140 \mu \mathrm{L})$. Purification by column chromatography $(10-50 \%$ EtOAc/heptane) afforded the title compound as a brown oil (Run 1: $125 \mathrm{mg}, 70 \%$ yield; Run 2: $127 \mathrm{mg}, 68 \%$ yield). ${ }^{1} \mathrm{H}$ NMR (400 $\mathrm{MHz}$, Chloroform-d) $\delta 6.71-6.66(\mathrm{~m}, 2 \mathrm{H}), 6.60(\mathrm{dd}, J=8.5,6.8 \mathrm{~Hz}, 1 \mathrm{H}), 4.15(\mathrm{dd}, J=10.8,2.8 \mathrm{~Hz}$, $1 \mathrm{H}), 4.04(\mathrm{dd}, \mathrm{J}=10.8,5.4 \mathrm{~Hz}, 1 \mathrm{H}), 3.81(\mathrm{br} \mathrm{s}, 1 \mathrm{H}), 3.70(\mathrm{dd}, J=10.5,5.0 \mathrm{~Hz}, 1 \mathrm{H}), 3.66-3.52(\mathrm{~m}$, $2 \mathrm{H}), 2.38(\mathrm{br} \mathrm{s}, 1 \mathrm{H}), 2.12(\mathrm{~s}, 3 \mathrm{H}) .{ }^{13} \mathrm{C}\left\{{ }^{1} \mathrm{H}\right\}$ NMR $(101 \mathrm{MHz}$, Chloroform-d) $\delta 143.5,130.7,123.8,123.0$, 118.3, 114.6, 65.5, 62.7, 51.4, 16.8. HRMS (ESI-TOF) $\mathrm{m} / \mathrm{z}$ : $[\mathrm{M}+\mathrm{H}]^{+}$Calcd for $\mathrm{C}_{10} \mathrm{H}_{14} \mathrm{NO}_{2} 180.1025$; Found 180.1030 .<smiles>CS(=O)(=O)CC(CO)Nc1ccccc1S(C)(=O)=O</smiles>

(4-(Methylsulfonyl)-1,2,3,4-tetrahydroquinoxalin-2-yl)methanol (20). Prepared following General Procedure 1 using $\mathrm{N}$-(2-bromophenyl)methanesulfonamide (Run 1: $265 \mathrm{mg}$; Run 2: $253 \mathrm{mg}$ ) and 3-oxetanamine $(140 \mu \mathrm{L})$. Purification by column chromatography twice (40-100\% EtOAc/heptane) afforded the title compound as a light pink solid (Run 1: $193 \mathrm{mg}, 75 \%$ yield; Run 2: $196 \mathrm{mg}, 80 \%$ yield). ${ }^{1} \mathrm{H}$ NMR $(400 \mathrm{MHz}$, Chloroform- $d$ ) $\delta 7.46$ (dd, $J=8.2,1.2 \mathrm{~Hz}, 1 \mathrm{H}), 7.04-6.96(\mathrm{~m}, 1 \mathrm{H}), 6.75-$ $6.67(\mathrm{~m}, 1 \mathrm{H}), 6.64(\mathrm{dd}, J=8.0,1.4 \mathrm{~Hz}, 1 \mathrm{H}), 4.37(\mathrm{~s}, 1 \mathrm{H}), 4.09-3.96(\mathrm{~m}, 1 \mathrm{H}), 3.84-3.75(\mathrm{~m}, 1 \mathrm{H})$, $3.71-3.53(\mathrm{~m}, 2 \mathrm{H}), 3.40(\mathrm{dd}, J=13.6,8.1 \mathrm{~Hz}, 1 \mathrm{H}), 2.96(\mathrm{~s}, 3 \mathrm{H}), 1.93-1.83(\mathrm{~m}, 1 \mathrm{H}) .{ }^{13} \mathrm{C}\left\{{ }^{1} \mathrm{H}\right\} \mathrm{NMR}$ (101 MHz, Chloroform-d) $\delta 136.9,126.3,123.6,122.5,117.8,115.2,63.6,51.3,45.1,39.6$. HRMS (ESI-TOF) m/z: [M-H]- Calcd for $\mathrm{C}_{10} \mathrm{H}_{13} \mathrm{~N}_{2} \mathrm{O}_{3} \mathrm{~S}$ 241.0647; Found 241.0640.<smiles>CN1c2cc3ccccc3cc2OCC1CO</smiles>

(4-Methyl-3,4-dihydro-2H-naphtho[2,3-b][1,4]oxazin-3-yl)methanol (21). Prepared following General Procedure 1 using 3-bromo-2-naphthol (Run 1: $228 \mathrm{mg}$; Run 2: $226 \mathrm{mg}$ ) and $\mathrm{N}$-methyloxetan3-amine $(180 \mu \mathrm{L})$. Purification by column chromatography $(20-60 \%$ EtOAc/heptane) afforded the title compound as a brown oil (Run 1: $100 \mathrm{mg}, 43 \%$ yield; Run 2: $98 \mathrm{mg}, 42 \%$ yield). ${ }^{1} \mathrm{H}$ NMR (400 MHz, Chloroform-d) $\delta 7.58(\mathrm{t}, J=7.2 \mathrm{~Hz}, 2 \mathrm{H}), 7.28-7.22(\mathrm{~m}, 1 \mathrm{H}), 7.20-7.13(\mathrm{~m}, 2 \mathrm{H}), 6.81(\mathrm{~s}, 1 \mathrm{H}), 4.45$ (dd, $J=11.0,1.9 \mathrm{~Hz}, 1 \mathrm{H}), 4.13(\mathrm{dd}, J=11.0,2.7 \mathrm{~Hz}, 1 \mathrm{H}), 3.85-3.76(\mathrm{~m}, 2 \mathrm{H}), 3.46-3.38(\mathrm{~m}, 1 \mathrm{H})$, $3.12(\mathrm{~s}, 3 \mathrm{H}), 1.75(\mathrm{br} \mathrm{s}, 1 \mathrm{H}) .{ }^{13} \mathrm{C}\left\{{ }^{1} \mathrm{H}\right\} \mathrm{NMR}(101 \mathrm{MHz}$, Chloroform-d) $\delta 144.5,135.6,130.8,127.2$, 126.2, 125.4, 124.2, 122.4, 111.3, 105.2, 64.4, 60.5, 58.5, 37.8. HRMS (ESI-TOF) m/z: $[\mathrm{M}+\mathrm{H}]^{+}$Calcd for $\mathrm{C}_{14} \mathrm{H}_{16} \mathrm{NO}_{2} 230.1181$; Found 230.1192 .<smiles>CC1(CO)COc2cc3ccccc3cc2N1</smiles>

(3-Methyl-3,4-dihydro-2H-naphtho[2,3-b][1,4]oxazin-3-yl)methanol (22). Prepared following General Procedure 1 using 3-bromo-2-naphthol (Run 1: $221 \mathrm{mg}$; Run 2: $226 \mathrm{mg}$ ) and 3-methyloxetan3 -amine $(90 \mu \mathrm{L})$. Purification by column chromatography (10-60\% EtOAc/heptane) afforded the title compound as an orange solid (Run 1: $131 \mathrm{mg}, 58 \%$ yield; Run 2: $137 \mathrm{mg}, 59 \%$ yield). ${ }^{1} \mathrm{H}$ NMR (400 $\mathrm{MHz}$, Chloroform- $d) \delta 7.59(\mathrm{~d}, J=8.0 \mathrm{~Hz}, 1 \mathrm{H}), 7.53(\mathrm{~d}, J=8.0 \mathrm{~Hz}, 1 \mathrm{H}), 7.25-7.16(\mathrm{~m}, 3 \mathrm{H}), 6.91(\mathrm{~s}$, 
$1 \mathrm{H}), 4.21(\mathrm{~d}, J=10.8 \mathrm{~Hz}, 1 \mathrm{H}), 4.11(\mathrm{~s}, 1 \mathrm{H}), 3.90(\mathrm{~d}, J=10.8 \mathrm{~Hz}, 1 \mathrm{H}), 3.68-3.55(\mathrm{~m}, 2 \mathrm{H}), 1.83(\mathrm{br}$ $\mathrm{s}, 1 \mathrm{H}), 1.27(\mathrm{~s}, 3 \mathrm{H}) .{ }^{13} \mathrm{C}\left\{{ }^{1} \mathrm{H}\right\}$ NMR $(101 \mathrm{MHz}$, Chloroform-d) $\delta 144.0,133.3,130.3,128.2,126.4,125.2$, 124.2, 122.8, 111.9, 109.3, 70.3, 66.4, 51.8, 21.0. HRMS (ESI-TOF) $\mathrm{m} / \mathrm{z}:[\mathrm{M}+\mathrm{H}]^{+}$Calcd for $\mathrm{C}_{14} \mathrm{H}_{16} \mathrm{NO}_{2}$ 230.1181; Found 230.1192.

General Procedure 2: To a 2.0 - $5.0 \mathrm{~mL}$ Biotage microwave vial was added aryl halide $(0.36$ $\mathrm{mmol})$, sulfonamide $(0.72 \mathrm{mmol})$, BrettPhos Pd G3 (16 mg, $0.018 \mathrm{mmol})$, and $\mathrm{K}_{2} \mathrm{CO}_{3}(173$ $\mathrm{mg}, 1.25 \mathrm{mmol})$. The vial was capped then evacuated and backfilled with Argon three times. 1 ,4-Dioxane $(1.0 \mathrm{~mL})$ was added and the vial placed in a heating block at $120^{\circ} \mathrm{C}$. The reaction mixture was stirred overnight (16h) then diluted with EtOAc and filtered over a pad of silica and concentrated. Purification by column chromatograpy (EtOAc/heptane) or reverse phase HPLC (Kinetex 5 um EVO C18 100 A, $100 \times 30$ mm, 10-30\% MeCN/10 mM NH ${ }_{4} \mathrm{OH}$ in water) afforded the title compounds.<smiles>[R5]N1CC(CO)Oc2cc3ccccc3cc21</smiles>

(4-(Methylsulfonyl)-3,4-dihydro-2H-naphtho[2,3-b][1,4]oxazin-2-yl)methanol

(25). Prepared following General Procedure 2 using 3-((3-bromonaphthalen-2-yl)oxy)oxetane (Run 1: 100 $\mathrm{mg}$; Run 2: $105 \mathrm{mg}$ ) and methanesulfonamide $(70 \mathrm{mg})$. Purification by column chromatography (20$60 \%$ EtOAc/heptane) afforded the title compound as an off-white solid (Run 1: $103.9 \mathrm{mg}, 99 \%$ yield; Run 2: $108.6 \mathrm{mg}, 99 \%$ yield). ${ }^{1} \mathrm{H}$ NMR $(400 \mathrm{MHz}$, Chloroform- $d) \delta 8.14(\mathrm{~s}, 1 \mathrm{H}), 7.76(\mathrm{~d}, \mathrm{~J}=8.0 \mathrm{~Hz}$, $1 \mathrm{H}), 7.67(\mathrm{~d}, \mathrm{~J}=8.0 \mathrm{~Hz}, 1 \mathrm{H}), 7.43-7.33(\mathrm{~m}, 3 \mathrm{H}), 4.39-4.28(\mathrm{~m}, 2 \mathrm{H}), 4.03-3.94(\mathrm{~m}, 1 \mathrm{H}), 3.94-$ $3.84(\mathrm{~m}, 1 \mathrm{H}), 3.68-3.57(\mathrm{~m}, 1 \mathrm{H}), 3.04(\mathrm{~s}, 3 \mathrm{H}), 2.01-1.94(\mathrm{~m}, 1 \mathrm{H}) .{ }^{13} \mathrm{C}\left\{{ }^{1} \mathrm{H}\right\} \mathrm{NMR}(101 \mathrm{MHz}$, Chloroform-d) $\delta 145.0,131.3,128.9,127.6,126.1,126.0,125.0,124.7,118.8,113.2,74.1,62.6$, 45.6, 38.3. HRMS (ESI-TOF) m/z: $[\mathrm{M}+\mathrm{H}]+$ Calcd for $\mathrm{C}_{14} \mathrm{H}_{16} \mathrm{NO}_{4} \mathrm{~S} 294.0800$; found 294.0813.<smiles>[R15]N1CC(CO)Oc2ccc(OC)cc21</smiles>

(6-Methoxy-4-(methylsulfonyl)-3,4-dihydro-2H-benzo[b][1,4]oxazin-2-yl)methanol (26). Prepared following General Procedure 2 using 3-(2-bromo-4-methoxyphenoxy)oxetane (Run 1: 92.8 $\mathrm{mg}$; Run 2: $99.0 \mathrm{mg}$ ) and methanesulfonamide $(70 \mathrm{mg})$. Purification by silica gel column chromatography (20-60\% EtOAc/heptane) afforded the title compound as a colorless oil which solidified upon standing to a pale yellow solid (Run $1: 84.7 \mathrm{mg}, 87 \%$ yield; Run $2: 91.4 \mathrm{mg}, 88 \%$ yield). ${ }^{1} \mathrm{H}$ NMR $(400 \mathrm{MHz}$, Chloroform-d) $\delta 7.28(\mathrm{~d}, J=2.9 \mathrm{~Hz}, 1 \mathrm{H}), 6.88(\mathrm{~d}, J=9.0 \mathrm{~Hz}, 1 \mathrm{H}), 6.66(\mathrm{dd}, J=9.0,2.9$ $\mathrm{Hz}, 1 \mathrm{H}), 4.23(\mathrm{dd}, J=13.7,2.5 \mathrm{~Hz}, 1 \mathrm{H}), 4.20-4.14(\mathrm{~m}, 1 \mathrm{H}), 3.95-3.87(\mathrm{~m}, 1 \mathrm{H}), 3.86-3.78(\mathrm{~m}$, $1 \mathrm{H}), 3.77(\mathrm{~s}, 3 \mathrm{H}), 3.45(\mathrm{dd}, J=13.7,9.3 \mathrm{~Hz}, 1 \mathrm{H}), 2.98(\mathrm{~s}, 3 \mathrm{H}), 1.99(\mathrm{t}, J=6.4 \mathrm{~Hz}, 1 \mathrm{H}) .{ }^{13} \mathrm{C}\left\{{ }^{1} \mathrm{H}\right\} \mathrm{NMR}$ (101 MHz, Chloroform-d) $\delta 154.0,140.1,124.2,118.2,112.0,106.9,73.4,62.6,55.8,45.5,38.5$. HRMS (ESI-TOF) m/z: [M-H]- Calcd for $\mathrm{C}_{11} \mathrm{H}_{14} \mathrm{NO}_{5} \mathrm{~S} 272.0593$; Found 272.0587.<smiles>[R15]N1CC(CO)Oc2cc(C(F)(F)F)ccc21</smiles>

(4-(Methylsulfonyl)-7-(trifluoromethyl)-3,4-dihydro-2H-benzo[b][1,4]oxazin-2-

yl)methanol (27). Prepared following General Procedure 2 using 3-(2-bromo-5(trifluoromethyl)phenoxy)oxetane (Run 1: $107.1 \mathrm{mg}$; Run 2: $111.5 \mathrm{mg}$ ) and methanesulfonamide (70 $\mathrm{mg}$ ). Purification by silica gel column chromatography (20-60\% EtOAc/heptane) afforded the title 
compound as a colorless oil (Run 1: $108.1 \mathrm{mg}, 96 \%$ yield; Run 2: $112.5 \mathrm{mg}, 96 \%$ yield). ${ }^{1} \mathrm{H}$ NMR (400 $\mathrm{MHz}$, Chloroform-d) $\delta 7.76(\mathrm{~d}, J=8.6 \mathrm{~Hz}, 1 \mathrm{H}), 7.33-7.13(\mathrm{~m}, 2 \mathrm{H}), 4.34-4.20(\mathrm{~m}, 2 \mathrm{H}), 4.01-3.81$ $(\mathrm{m}, 2 \mathrm{H}), 3.59-3.45(\mathrm{~m}, 1 \mathrm{H}), 3.04(\mathrm{~s}, 3 \mathrm{H}), 2.18(\mathrm{t}, \mathrm{J}=6.0 \mathrm{~Hz}, 1 \mathrm{H}) .{ }^{13} \mathrm{C}\left\{{ }^{1} \mathrm{H}\right\} \mathrm{NMR}(101 \mathrm{MHz}, \mathrm{CDCl} 3$, $298 \mathrm{~K}) \delta(\mathrm{ppm})=145.8,127.5(\mathrm{q}, J=33.5 \mathrm{~Hz}), 127.2,123.6(\mathrm{q}, J=272.2 \mathrm{~Hz}), 121.7,118.1(\mathrm{q}, J=$ $3.7 \mathrm{~Hz}), 115.3(\mathrm{q}, J=3.7 \mathrm{~Hz}), 74.1,62.1,45.0,39.1 .{ }^{19} \mathrm{~F}\left\{{ }^{1} \mathrm{H}\right\} \mathrm{NMR}(376 \mathrm{MHz}$, Chloroform- $d) \delta-62.5$. HRMS (ESI-TOF) $\mathrm{m} / \mathrm{z}$ : [M-H] ${ }^{-}$Calcd for $\mathrm{C}_{11} \mathrm{H}_{11} \mathrm{~F}_{3} \mathrm{NO}_{4} \mathrm{~S} 310.0361$ found 310.0359 .<smiles>[R15]CC(CO)Oc1ccc([N+](=O)[O-])cc1S</smiles>

(4-(Methylsulfonyl)-6-nitro-3,4-dihydro-2H-benzo[b][1,4]oxazin-2-yl)methanol

(28).

Prepared following General Procedure 2 using 3-(2-bromo-4-nitrophenoxy)oxetane (Run 1: 98.7 mg; Run 2: $100.3 \mathrm{mg}$ ) and methanesulfonamide $(70 \mathrm{mg})$. Purification by silica gel column chromatography (50-100\% EtOAc/heptane) afforded the title compound as a yellow solid (Run 1: $89.8 \mathrm{mg}, 87 \%$ yield; Run 2: $100.7 \mathrm{mg}, 95 \%$ yield). ${ }^{1} \mathrm{H}$ NMR $(400 \mathrm{MHz}$, Chloroform- $d$ ) $\delta 8.54(\mathrm{~d}, \mathrm{~J}=2.6 \mathrm{~Hz}, 1 \mathrm{H}), 7.99$ (dd, $\mathrm{J}$ $=9.1,2.6 \mathrm{~Hz}, 1 \mathrm{H}), 7.06(\mathrm{~d}, J=9.1 \mathrm{~Hz}, 1 \mathrm{H}), 4.41-4.33(\mathrm{~m}, 1 \mathrm{H}), 4.23(\mathrm{dd}, J=14.0,2.6 \mathrm{~Hz}, 1 \mathrm{H}), 4.01$ - $3.86(\mathrm{~m}, 2 \mathrm{H}), 3.57(\mathrm{dd}, J=14.0,8.6 \mathrm{~Hz}, 1 \mathrm{H}), 3.13(\mathrm{~s}, 3 \mathrm{H}), 1.99-1.90(\mathrm{~m}, 1 \mathrm{H}) .{ }^{13} \mathrm{C}\left\{{ }^{1} \mathrm{H}\right\} \mathrm{NMR}(101$ $\mathrm{MHz}$, DMSO-d 6 ) $\delta 152.32,140.99,124.72,121.25,118.64,118.16,75.33,61.07,44.84,38.90$. HRMS (ESI-TOF) m/z: [M-H] $]^{-}$Calcd for $\mathrm{C}_{10} \mathrm{H}_{11} \mathrm{~N}_{2} \mathrm{O}_{6} \mathrm{~S} 287.0338$; found 287.0338.<smiles>[R5]N1CC(CO)Oc2c(Cl)cccc21</smiles>

(8-Chloro-4-(methylsulfonyl)-3,4-dihydro-2H-benzo[b][1,4]oxazin-2-yl)methanol

(29). Prepared following General Procedure 2 using 3-(2-bromo-6-chlorophenoxy)oxetane (Run 1: 95.0 mg; Run 2: $94.7 \mathrm{mg})$ and methanesulfonamide $(70 \mathrm{mg})$. Purification by silica gel column chromatography (20-60\% EtOAc/heptane) afforded the title compound as a white solid (Run 1: $63.4 \mathrm{mg}, 63 \%$ yield; Run 2: $59.2 \mathrm{mg}, 59 \%$ yield). ${ }^{1} \mathrm{H}$ NMR $(400 \mathrm{MHz}$, Chloroform- $d$ ) $\delta 7.57$ (dd, $J=8.4,1.4 \mathrm{~Hz}, 1 \mathrm{H}), 7.18$ (dd, $J$ $=8.0,1.4 \mathrm{~Hz}, 1 \mathrm{H}), 6.88(\mathrm{t}, J=8.2 \mathrm{~Hz}, 1 \mathrm{H}), 4.37-4.30(\mathrm{~m}, 1 \mathrm{H}), 4.25(\mathrm{dd}, J=14.0,2.7 \mathrm{~Hz}, 1 \mathrm{H}), 4.03$ - $3.96(\mathrm{~m}, 1 \mathrm{H}), 3.92-3.84(\mathrm{~m}, 1 \mathrm{H}), 3.52(\mathrm{dd}, J=14.0,9.2 \mathrm{~Hz}, 1 \mathrm{H}), 3.02(\mathrm{~s}, 3 \mathrm{H}), 2.03(\mathrm{dd}, J=7.1$, $6.3 \mathrm{~Hz}, 1 \mathrm{H}) .{ }^{13} \mathrm{C}\left\{{ }^{1} \mathrm{H}\right\} \mathrm{NMR}(101 \mathrm{MHz}$, Chloroform-d) $\delta 142.4,126.5,125.4,122.7,121.0,120.6,74.6$, $62.2,44.9,39.3$. HRMS (ESI-TOF) m/z: [M-H]- Calcd for $\mathrm{C}_{10} \mathrm{H}_{11} \mathrm{CINO}_{4} \mathrm{~S} 276.0097$; found 276.0090 .<smiles>[R5]N1CC(CO)Oc2cccnc21</smiles>

(4-(Methylsulfonyl)-3,4-dihydro-2H-pyrido[3,2-b][1,4]oxazin-2-yl)methanol (30). Prepared following General Procedure 2 using 2-chloro-3-(oxetan-3-yloxy)pyridine (Run 1: $68.0 \mathrm{mg}$; Run 2: 73.0 $\mathrm{mg}$ ) and methanesulfonamide (70 mg) except using $1 \mathrm{~mol} \%$ of BrettPhos Pd G3 (3.3 mg, $0.0037 \mathrm{mmol})$. Purification by reverse phase HPLC (Kinetex 5 um EVO C18 100 A, $100 \times 30$ mm, 10-30\% MeCN/10 mM $\mathrm{NH}_{4} \mathrm{OH}$ in water) afforded the title compound as a colorless oil (Run 1: $63.6 \mathrm{mg}, 71 \%$ yield; Run 2: 69.5 $\mathrm{mg}, 72 \%$ yield). ${ }^{1} \mathrm{H}$ NMR $(400 \mathrm{MHz}$, Chloroform- $d$ ) $\delta 8.01$ (dd, $J=4.7,1.5 \mathrm{~Hz}, 1 \mathrm{H}), 7.24$ (dd, $J=8.1$, $1.5 \mathrm{~Hz}, 1 \mathrm{H}), 6.99(\mathrm{dd}, J=8.1,4.7 \mathrm{~Hz}, 1 \mathrm{H}), 4.39-4.28(\mathrm{~m}, 1 \mathrm{H}), 4.23(\mathrm{dd}, J=13.5,2.5 \mathrm{~Hz}, 1 \mathrm{H}), 3.98$ - $3.81(\mathrm{~m}, 2 \mathrm{H}), 3.77(\mathrm{dd}, \mathrm{J}=13.5,7.5 \mathrm{~Hz}, 1 \mathrm{H}), 3.55(\mathrm{~s}, 3 \mathrm{H}), 2.03(\mathrm{t}, J=6.0 \mathrm{~Hz}, 1 \mathrm{H}) .{ }^{13} \mathrm{C}\left\{{ }^{1} \mathrm{H}\right\} \mathrm{NMR}$ (101 MHz, Chloroform-d) $\delta 140.8,140.1,139.4,124.7,120.1,74.8,61.9,43.8,43.4$. HRMS (ESI-TOF) $\mathrm{m} / \mathrm{z}:[\mathrm{M}+\mathrm{H}]^{+}$Calcd for $\mathrm{C}_{9} \mathrm{H}_{13} \mathrm{~N}_{2} \mathrm{O}_{4} \mathrm{~S} 245.0596$; Found 245.0609. 
<smiles>CC1(CO)CN(S(C)(=O)=O)c2cc([N+](=O)[O-])ccc2O1</smiles>

(2-Methyl-4-(methylsulfonyl)-6-nitro-3,4-dihydro-2H-benzo[b][1,4]oxazin-2-yl)methanol (31). Prepared following General Procedure 2 using 3-(2-bromo-4-nitrophenoxy)-3-methyloxetane (Run 1: $102.4 \mathrm{mg}$; Run 2: $101.3 \mathrm{mg}$ ). After reaction completion, the mixture was diluted with EtOAc and filtered over a pad of celite and concentrated. The crude material was dissolved in EtOAc and water was added. Extracted with EtOAc three times. The combined organics were washed with brine, dried over sodium sulfate, filtered and concentrated. Purification by silica gel column chromatography (20$60 \%$ EtOAc/heptane) afforded the title compound as a pale yellow solid (Run 1: $94.8 \mathrm{mg}, 88 \%$ yield; Run 2: $95.5 \mathrm{mg}, 90 \%$ yield). ${ }^{1} \mathrm{H}$ NMR (400 MHz, Chloroform- $d$ ) $\delta 8.43$ (d, $J=2.5 \mathrm{~Hz}, 1 \mathrm{H}$ ), 7.95 (dd, $J$ $=9.1,2.6 \mathrm{~Hz}, 1 \mathrm{H}), 7.01(\mathrm{~d}, J=9.1 \mathrm{~Hz}, 1 \mathrm{H}), 4.00(\mathrm{~d}, J=13.4 \mathrm{~Hz}, 1 \mathrm{H}), 3.77(\mathrm{dd}, J=12.0,5.5 \mathrm{~Hz}, 1 \mathrm{H})$, $3.64-3.53(\mathrm{~m}, 2 \mathrm{H}), 3.26(\mathrm{~s}, 3 \mathrm{H}), 2.24(\mathrm{dd}, \mathrm{J}=8.3,5.6 \mathrm{~Hz}, 1 \mathrm{H}), 1.43(\mathrm{~s}, 3 \mathrm{H}) .{ }^{13} \mathrm{C}\left\{{ }^{1} \mathrm{H}\right\} \mathrm{NMR}(101 \mathrm{MHz}$, DMSO-d6) $\delta 150.3,141.0,125.1,120.2,118.8,114.7,78.4,64.6,48.4,39.1,20.5$. HRMS (ESI-TOF) $\mathrm{m} / \mathrm{z}:[\mathrm{M}+\mathrm{H}]^{+}$Calcd for $\mathrm{C}_{11} \mathrm{H}_{15} \mathrm{~N}_{2} \mathrm{O}_{6} \mathrm{~S} 303.0651$; Found 303.0656.<smiles>OCC1CN([125I])c2cc3ccccc3cc2O1</smiles>

(4-Tosyl-3,4-dihydro-2H-naphtho[2,3-b][1,4]oxazin-2-yl)methanol (32). Prepared following General Procedure 2 using 3-((3-bromonaphthalen-2-yl)oxy)oxetane (Run 1: 101.0 mg; Run 2: 104.7 $\mathrm{mg})$ and $p$-toluenesulfonamide $(128 \mathrm{mg})$. Purification by column chromatography (0-60\% EtOAc/heptane) afforded the title compound with residual $p$-toluenesulfonamide impurities. Further purification by reverse phase HPLC (Kinetex 5 um EVO C18 100 A, $100 \times 30$ mm, 10-30\% MeCN/10 mM $\mathrm{NH}_{4} \mathrm{OH}$ in water) afforded the title compound as a white solid (Run 1: $109.6 \mathrm{mg}, 82 \%$ yield; Run 2: $111.0 \mathrm{mg}, 80 \%$ yield). ${ }^{1} \mathrm{H}$ NMR $(400 \mathrm{MHz}$, Chloroform- $d) \delta 8.31(\mathrm{~s}, 1 \mathrm{H}), 7.80(\mathrm{~d}, J=7.8 \mathrm{~Hz}, 1 \mathrm{H}), 7.63$ $(\mathrm{d}, J=7.9 \mathrm{~Hz}, 1 \mathrm{H}), 7.57(\mathrm{~d}, J=8.3 \mathrm{~Hz}, 2 \mathrm{H}), 7.43-7.32(\mathrm{~m}, 2 \mathrm{H}), 7.24(\mathrm{~s}, 1 \mathrm{H}), 7.20(\mathrm{~d}, J=8.0 \mathrm{~Hz}$, $2 \mathrm{H}), 4.38(\mathrm{dd}, J=14.2,2.3 \mathrm{~Hz}, 1 \mathrm{H}), 3.88-3.78(\mathrm{~m}, 1 \mathrm{H}), 3.77-3.67(\mathrm{~m}, 2 \mathrm{H}), 3.53(\mathrm{dd}, J=14.2,9.7$ $\mathrm{Hz}, 1 \mathrm{H}), 2.36(\mathrm{~s}, 3 \mathrm{H}), 1.82(\mathrm{t}, J=6.3 \mathrm{~Hz}, 1 \mathrm{H}) .{ }^{13} \mathrm{C}\left\{{ }^{1} \mathrm{H}\right\} \mathrm{NMR}(101 \mathrm{MHz}$, Chloroform-d) $\delta 145.5,144.4$, $135.8,131.7,130.0,128.7,127.9,127.1,126.1,126.0,124.5,124.4,121.6,112.5,72.5,62.8,45.7$, 21.6. HRMS (ESI-TOF) m/z: $[\mathrm{M}+\mathrm{H}]^{+}$Calcd for $\mathrm{C}_{20} \mathrm{H}_{20} \mathrm{NO}_{4} \mathrm{~S} 370.1113$; Found 370.1118.<smiles></smiles>

Methyl 2-(hydroxymethyl)-4-((4-nitrophenyl)sulfonyl)-3,4-dihydro-2Hbenzo[b][1,4] oxazine-7-carboxylate (33). Prepared following General Procedure 2 using methyl 4bromo-3-(oxetan-3-yloxy)benzoate (Run 1: $103.4 \mathrm{mg}$; Run 2: $98.7 \mathrm{mg}$ ) and 4-nitrobenzenesulfonamide (146 mg). Purification by column chromatography (10-40\% EtOAc/heptane) afforded the title compound as a yellow solid (Run 1: $126.1 \mathrm{mg}, 86 \%$ yield; Run 2: $121.1 \mathrm{mg}, 86 \%$ yield). ${ }^{1} \mathrm{H}$ NMR (400 MHz, DMSO$\left.d_{6}\right) \delta 8.39(\mathrm{~d}, J=8.9 \mathrm{~Hz}, 2 \mathrm{H}), 8.03(\mathrm{~d}, J=9.0 \mathrm{~Hz}, 2 \mathrm{H}), 7.83(\mathrm{~d}, J=8.7 \mathrm{~Hz}, 1 \mathrm{H}), 7.55(\mathrm{dd}, J=8.7,2.0$ $\mathrm{Hz}, 1 \mathrm{H}), 7.37(\mathrm{~d}, J=2.0 \mathrm{~Hz}, 1 \mathrm{H}), 5.10-5.02(\mathrm{~m}, 1 \mathrm{H}), 4.41(\mathrm{dd}, J=13.8,1.8 \mathrm{~Hz}, 1 \mathrm{H}), 3.82(\mathrm{~s}, 3 \mathrm{H})$, $3.63-3.44(\mathrm{~m}, 4 \mathrm{H}) .{ }^{13} \mathrm{C}\left\{{ }^{1} \mathrm{H}\right\} \operatorname{NMR}\left(101 \mathrm{MHz}, \mathrm{DMSO}-d_{6}\right) \delta 165.7,151.0,146.6,143.0,129.2,127.8$, 127.6, 125.6, 123.1, 122.1, 118.7, 73.8, 61.0, 52.7, 46.0. HRMS (ESI-TOF) m/z: [M-H]- Calcd for $\mathrm{C}_{17} \mathrm{H}_{15} \mathrm{~N}_{2} \mathrm{O}_{8} \mathrm{~S}$ 407.0549; Found 407.0556. 
5. NMR Spectra 

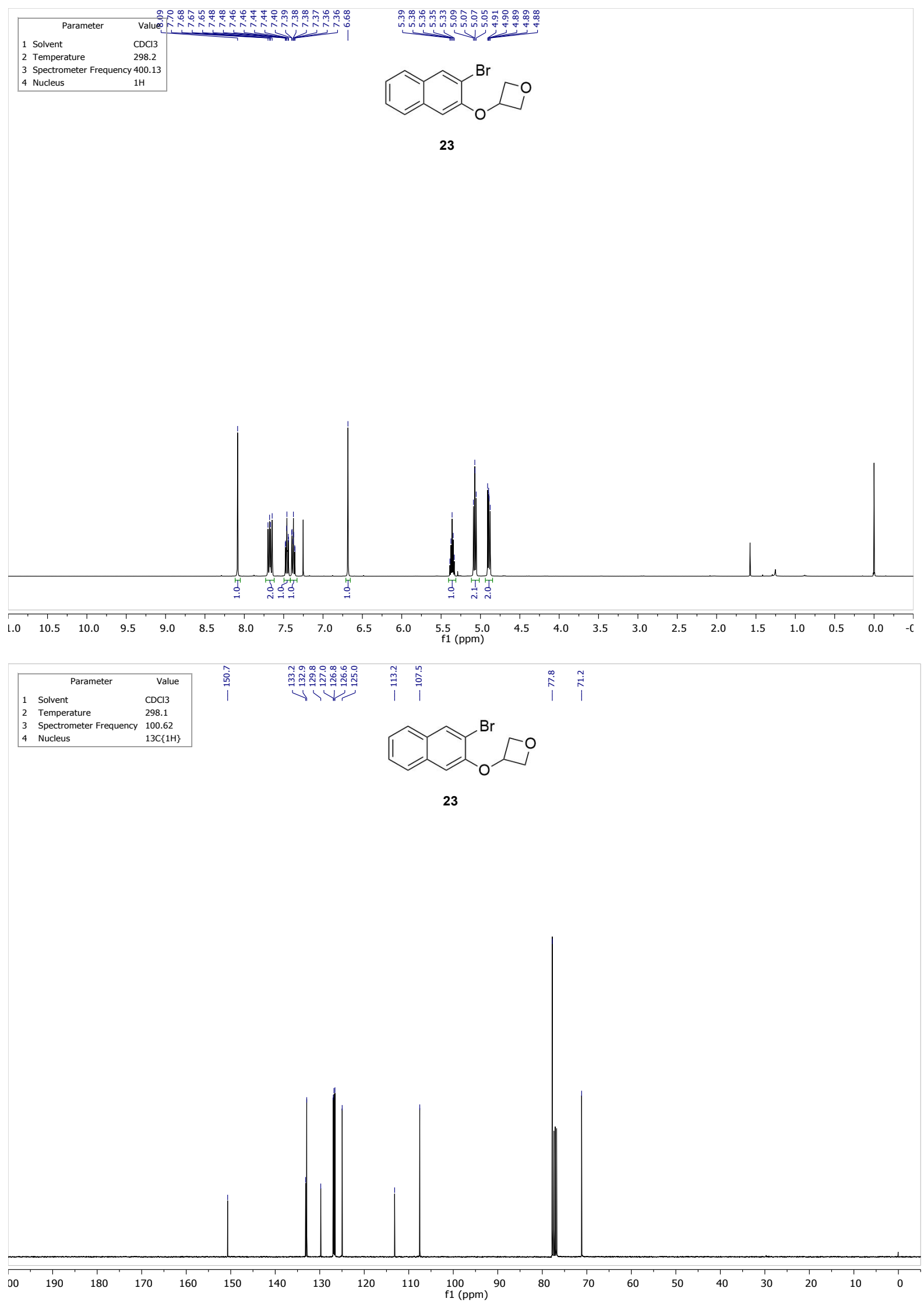


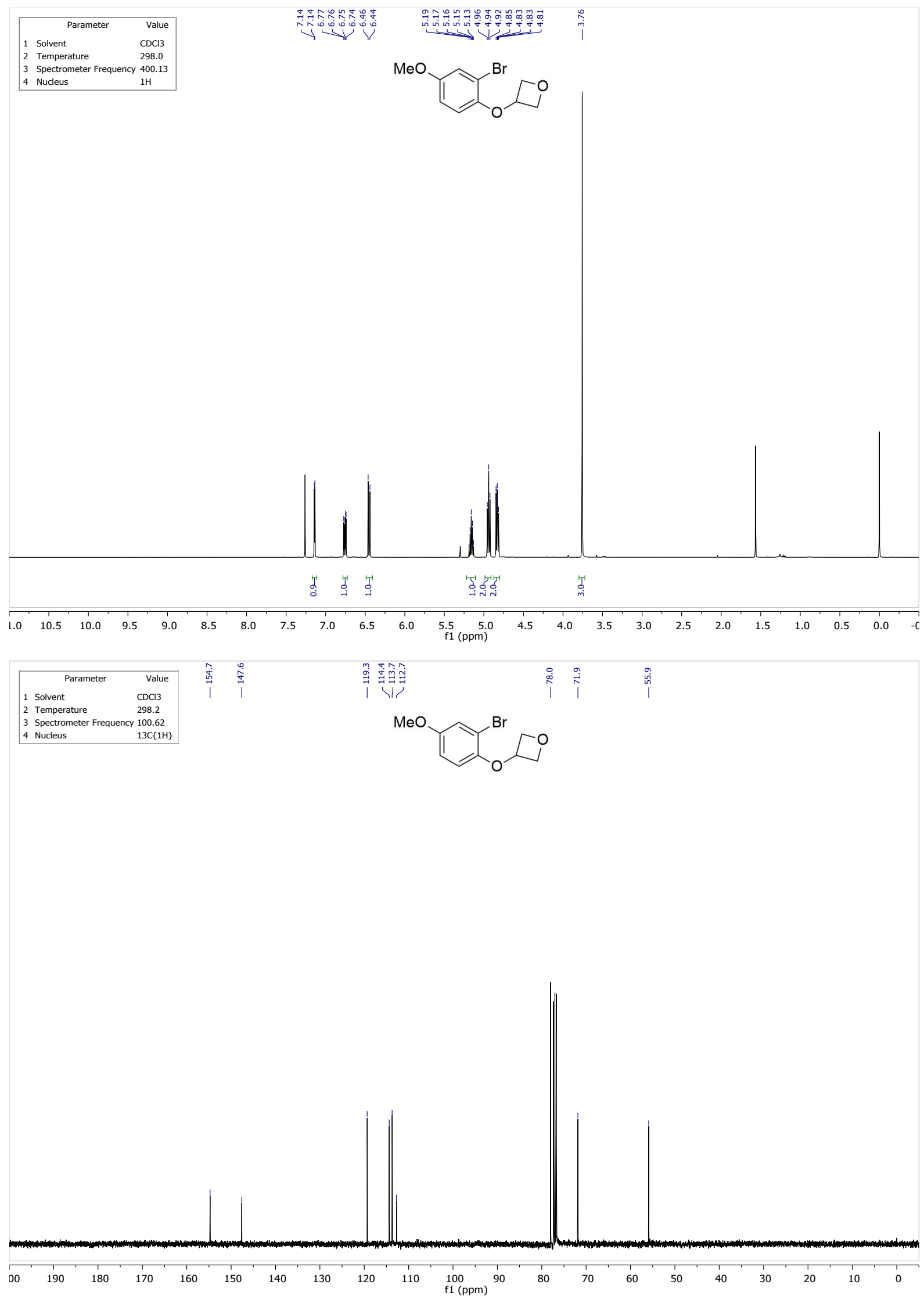




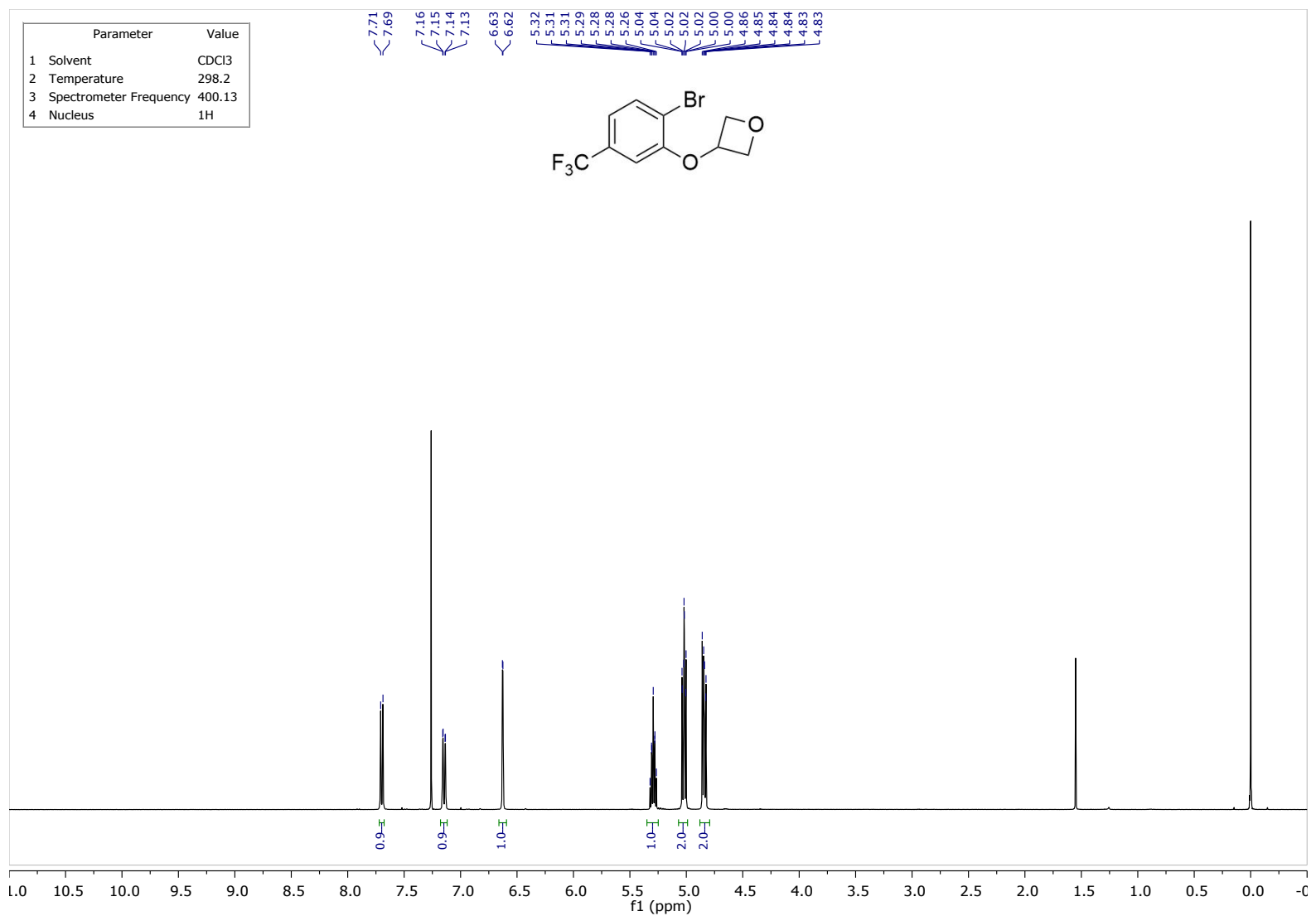




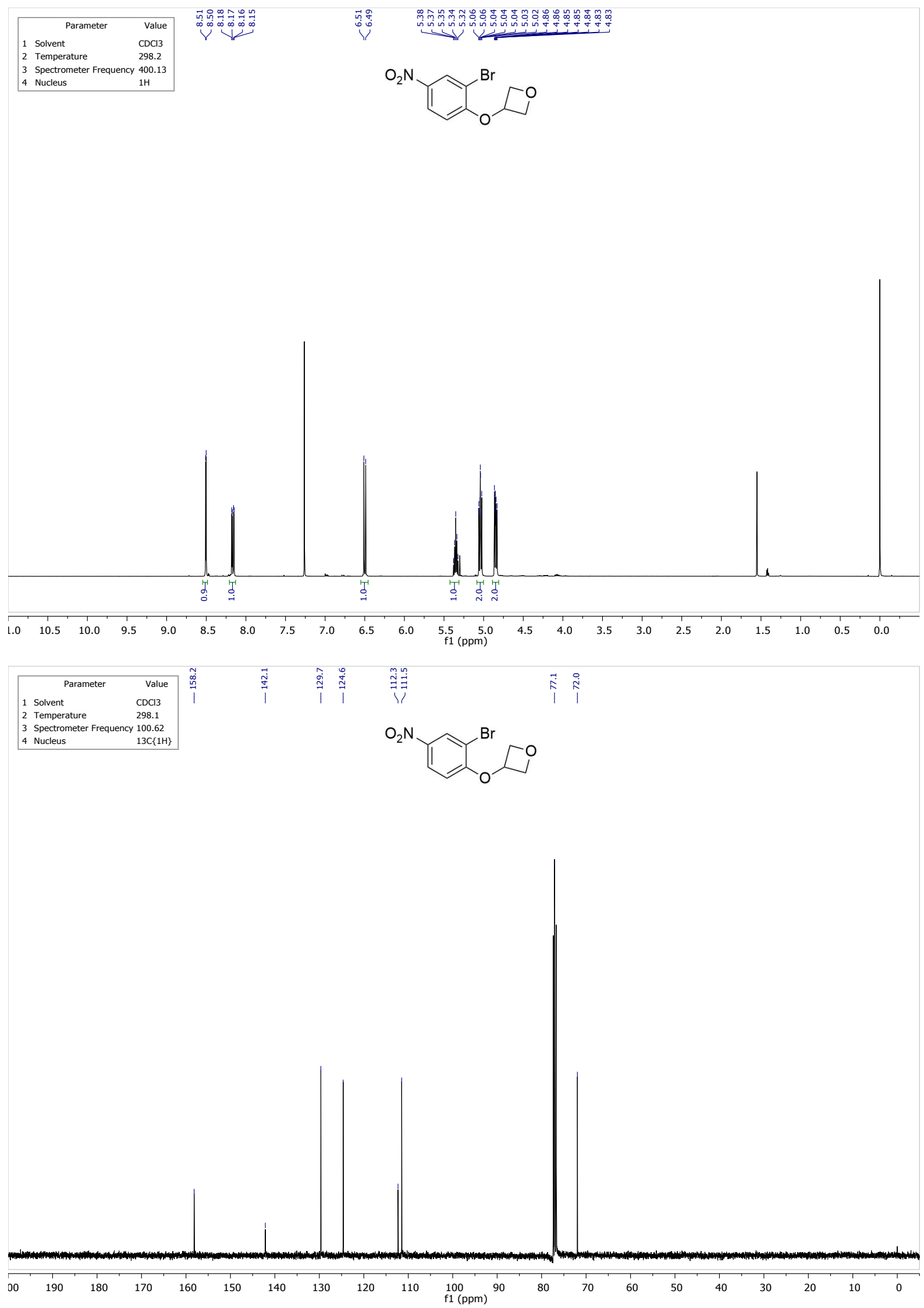




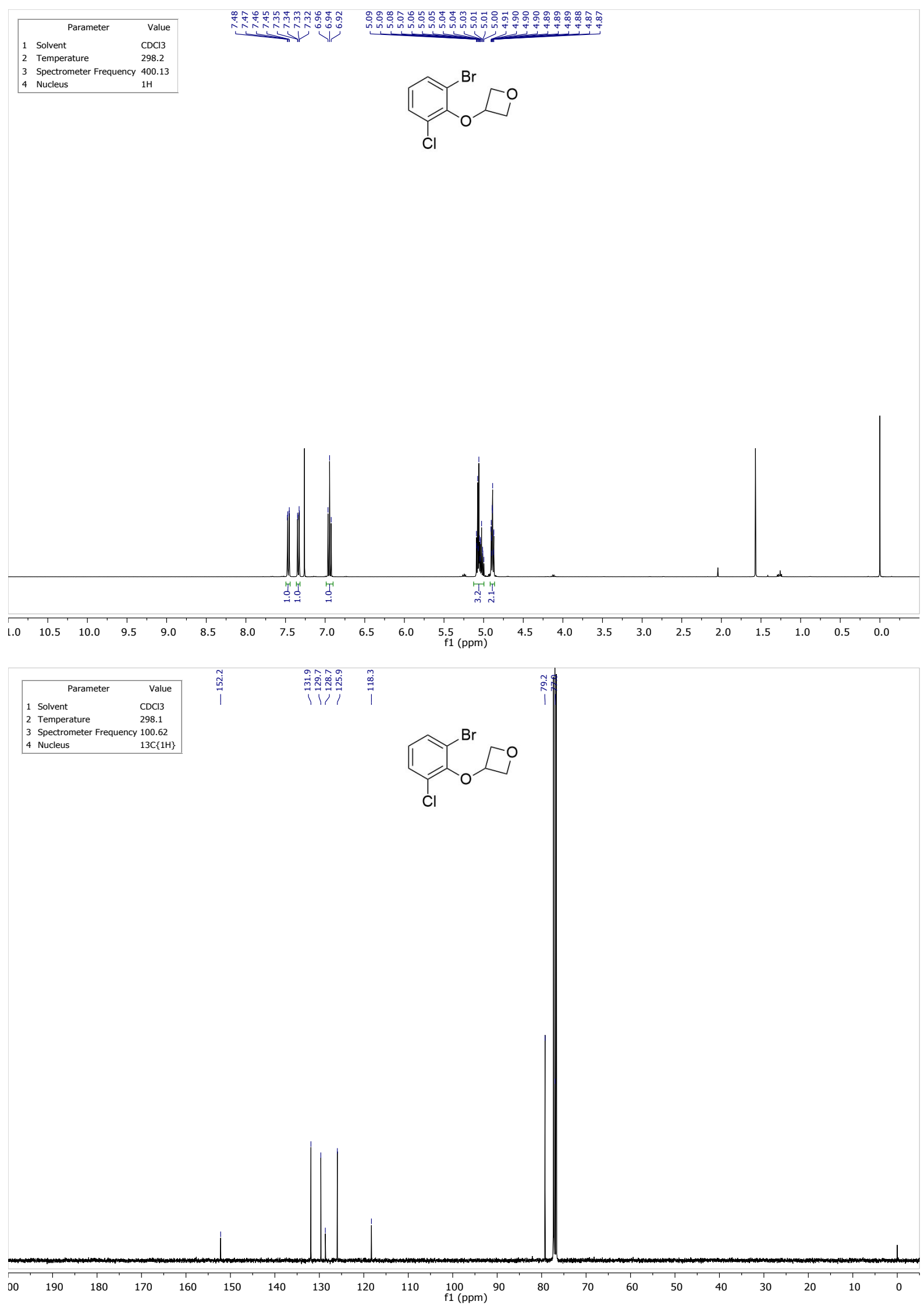



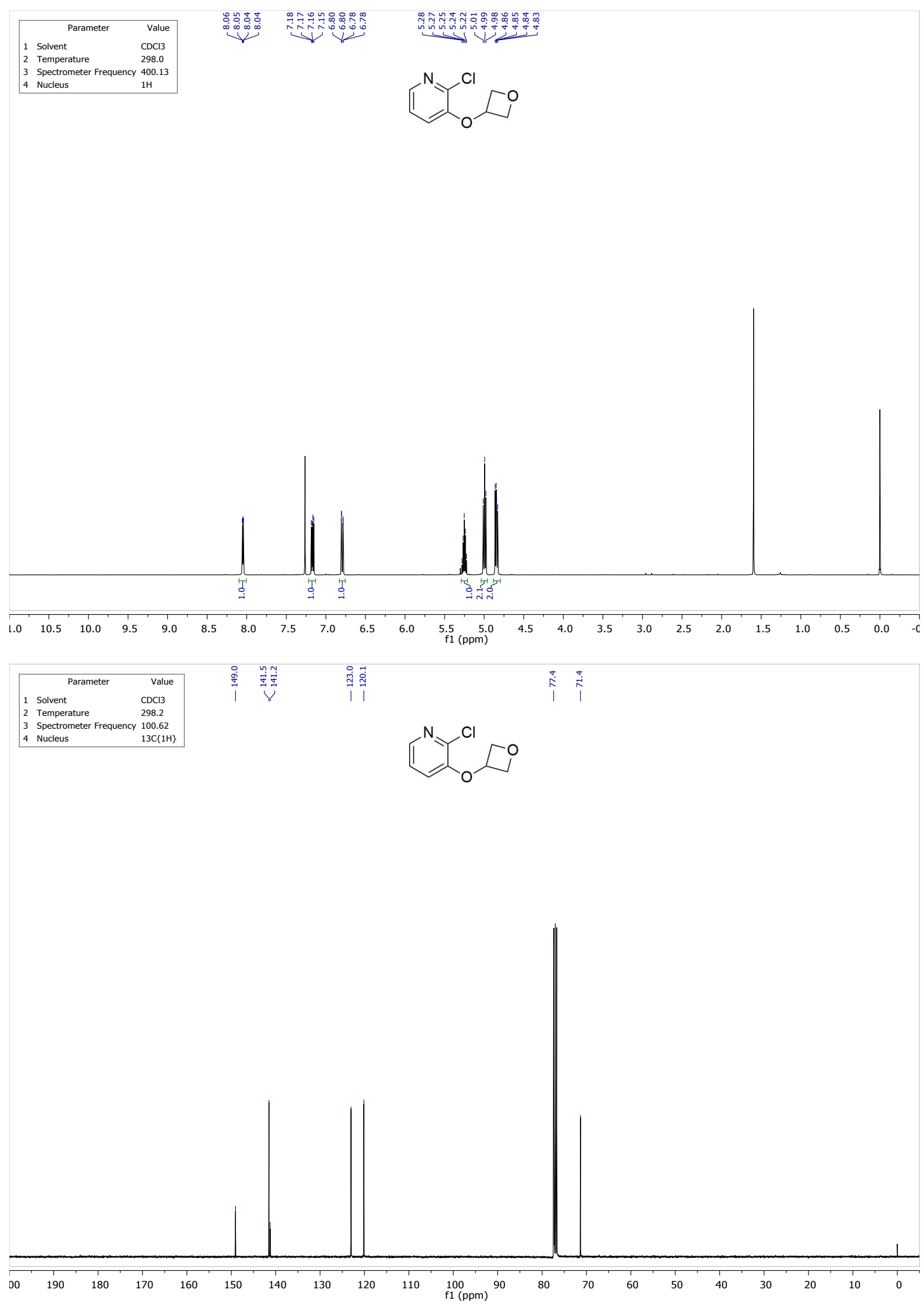


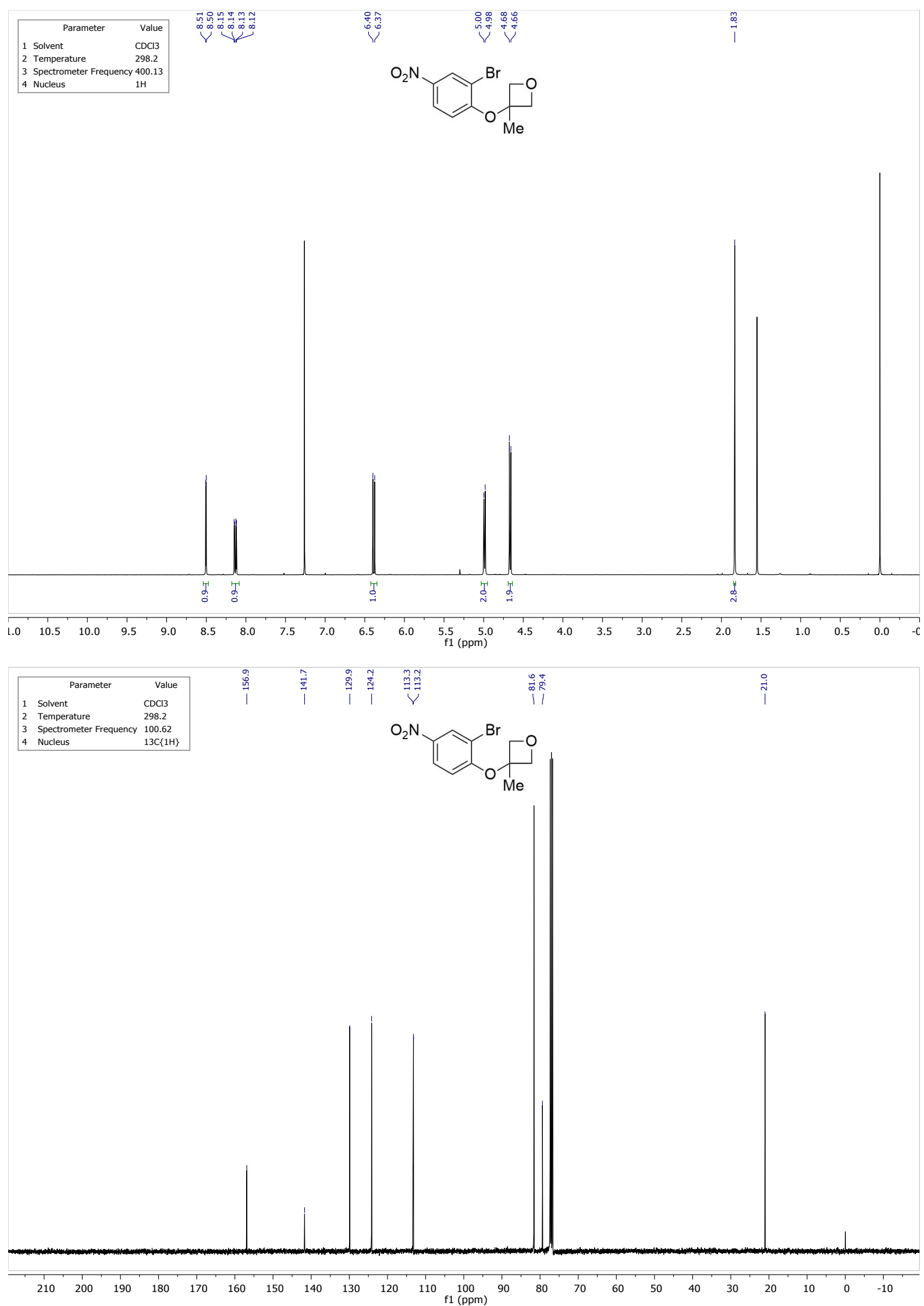



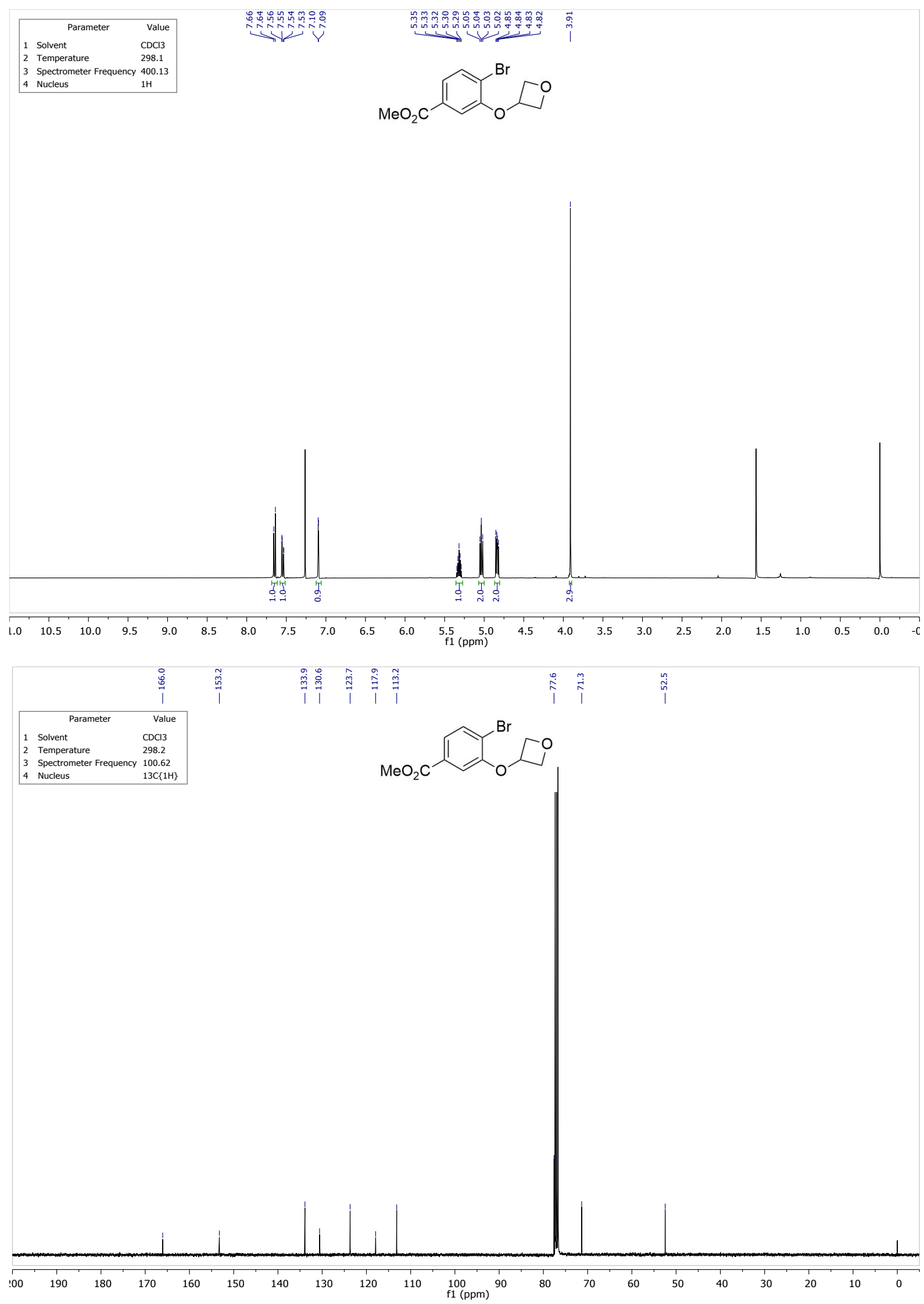

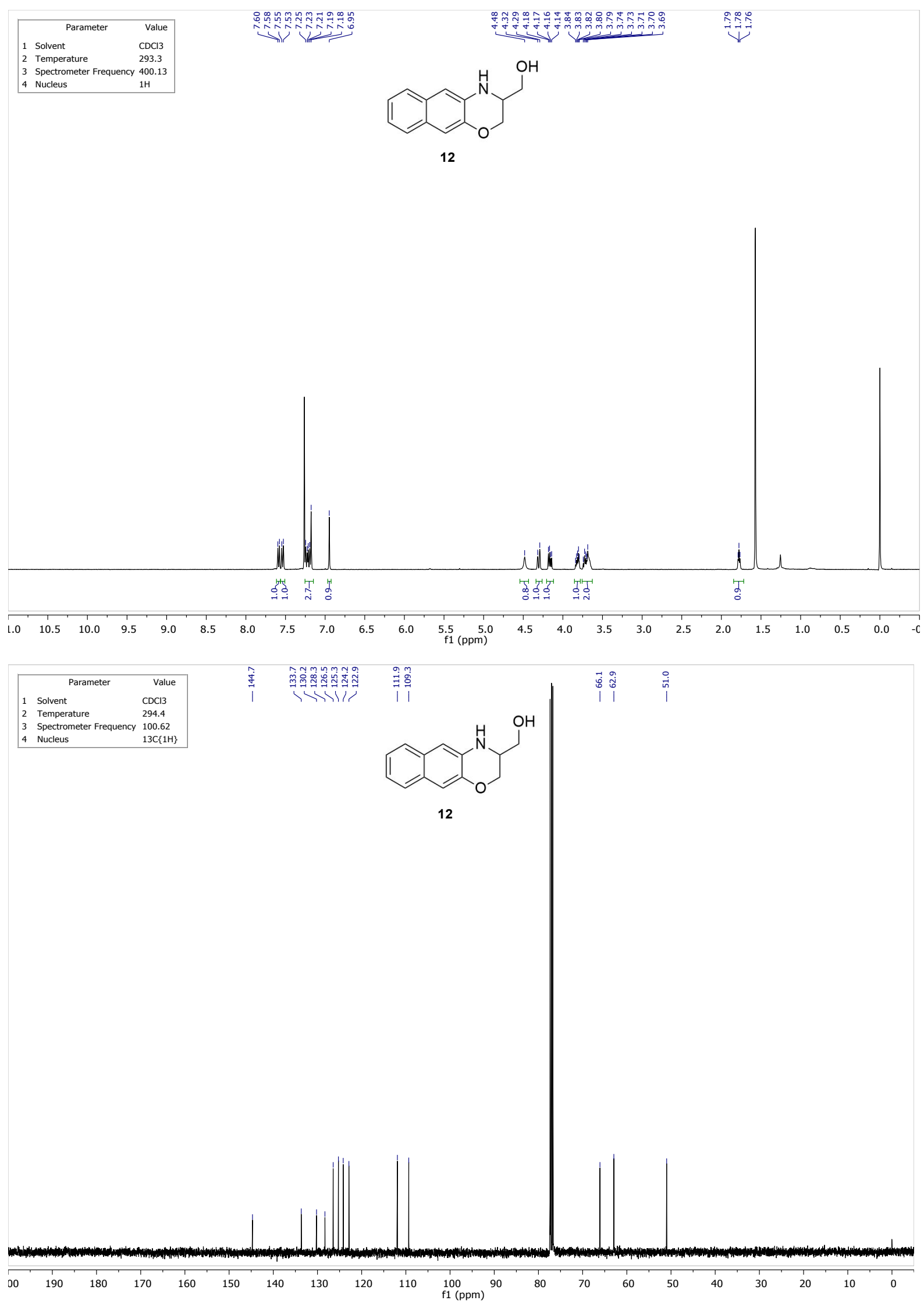


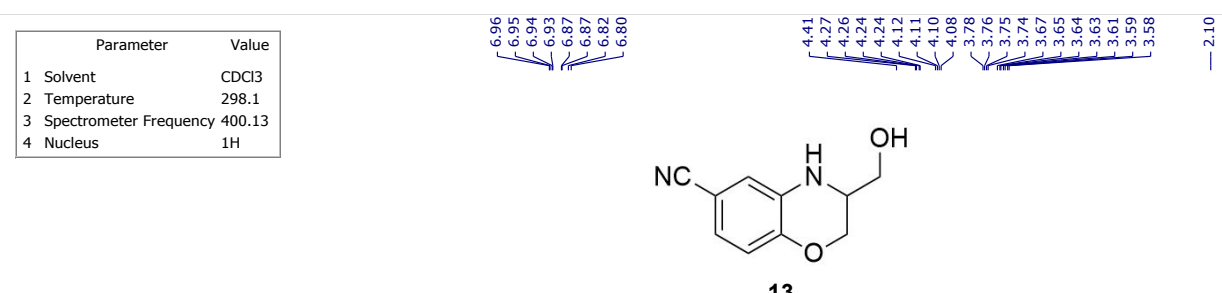

13
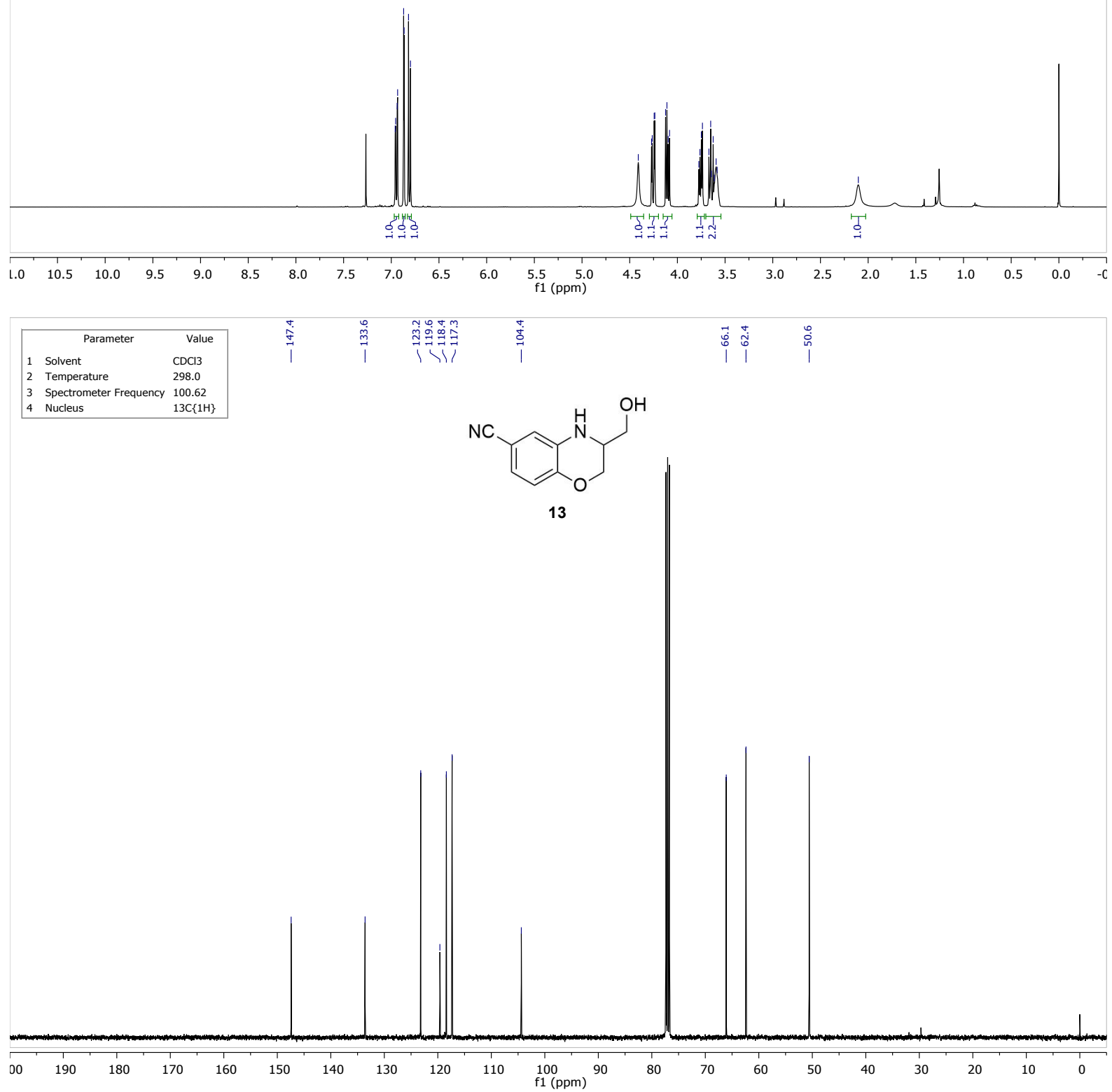

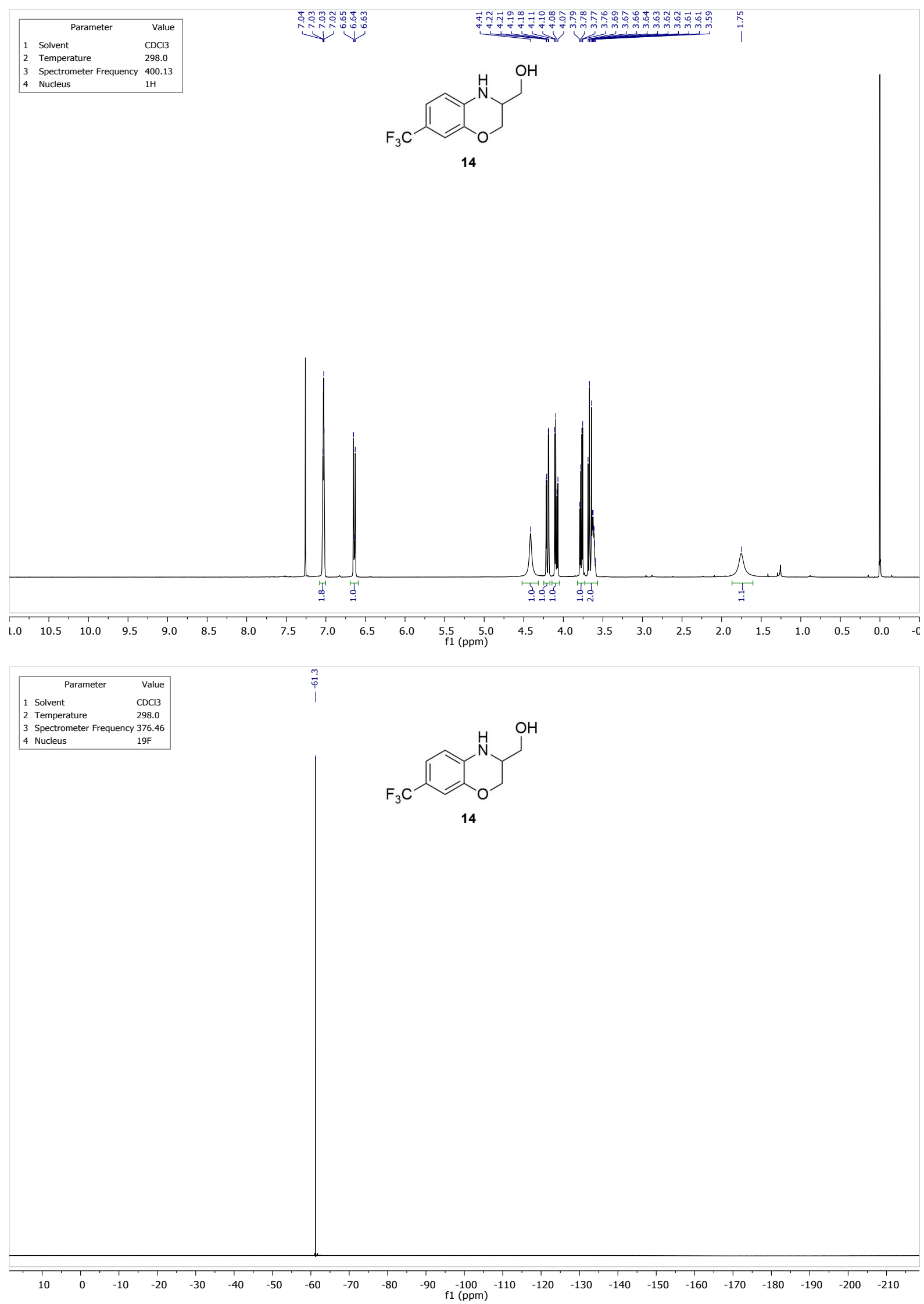


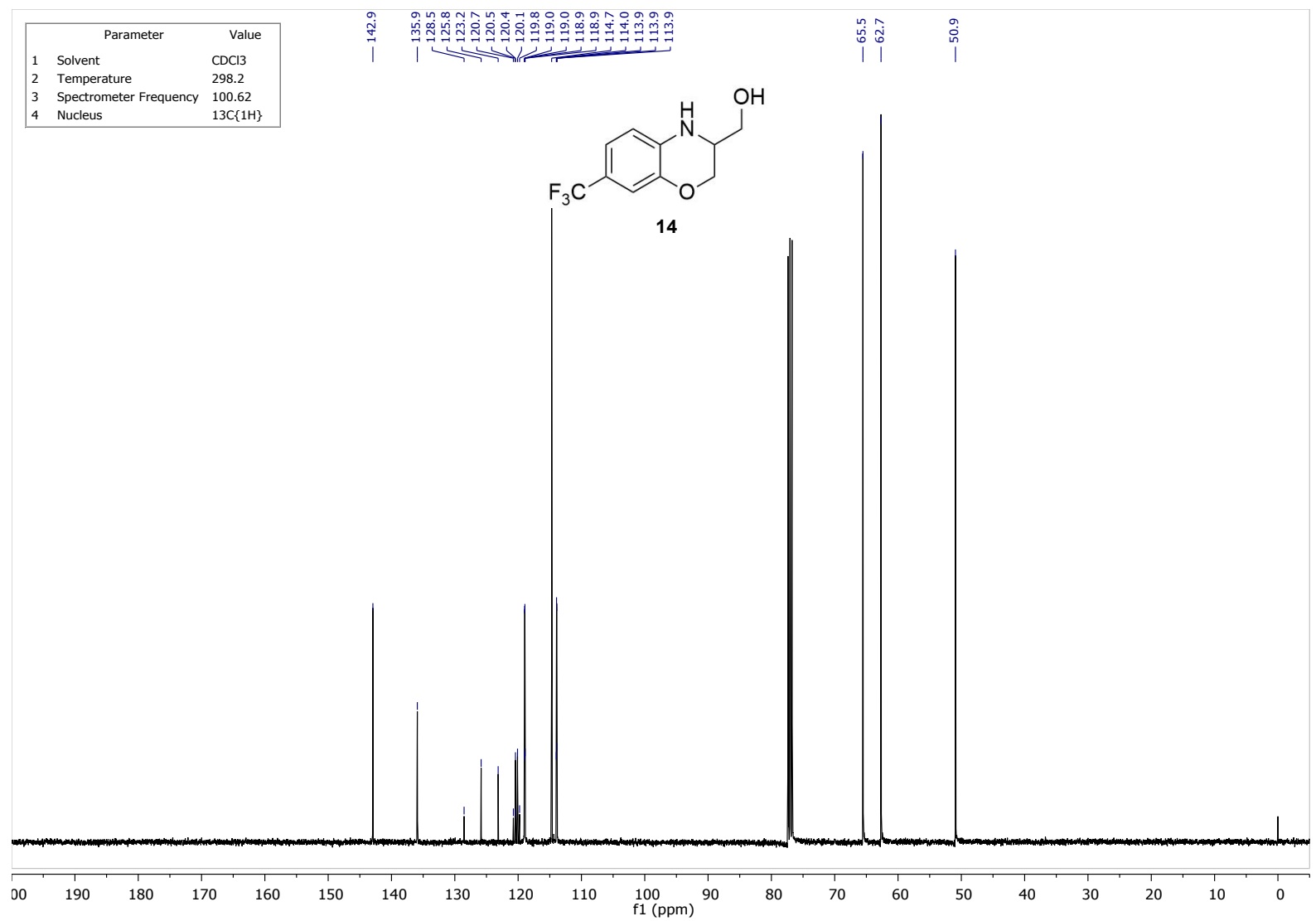




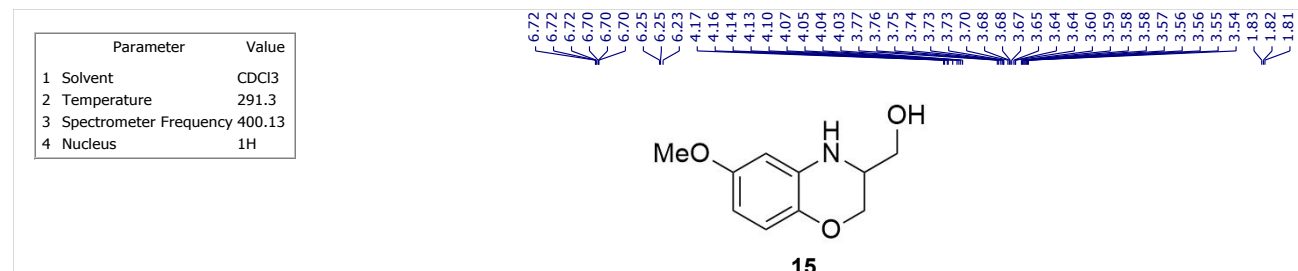

15
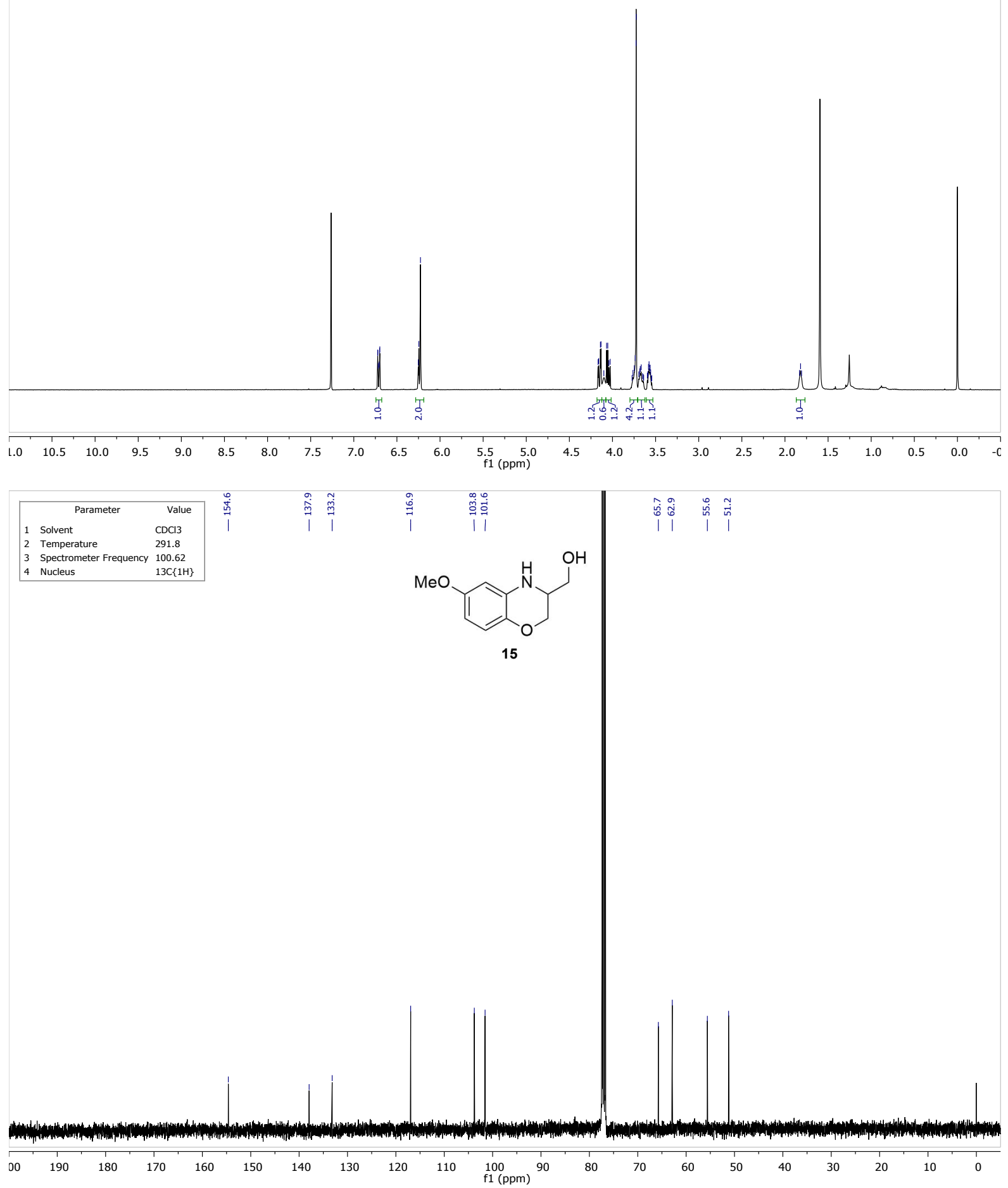


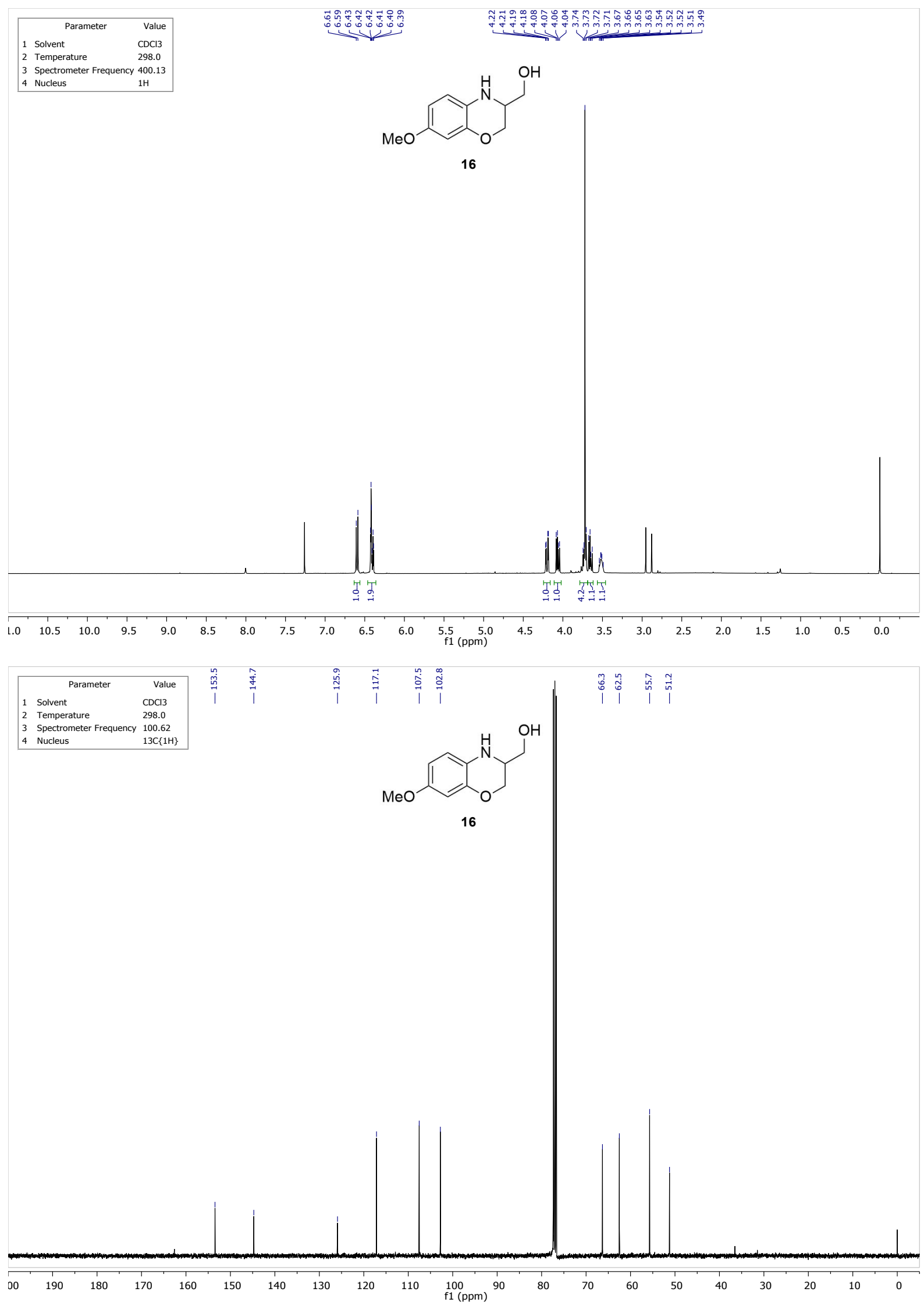



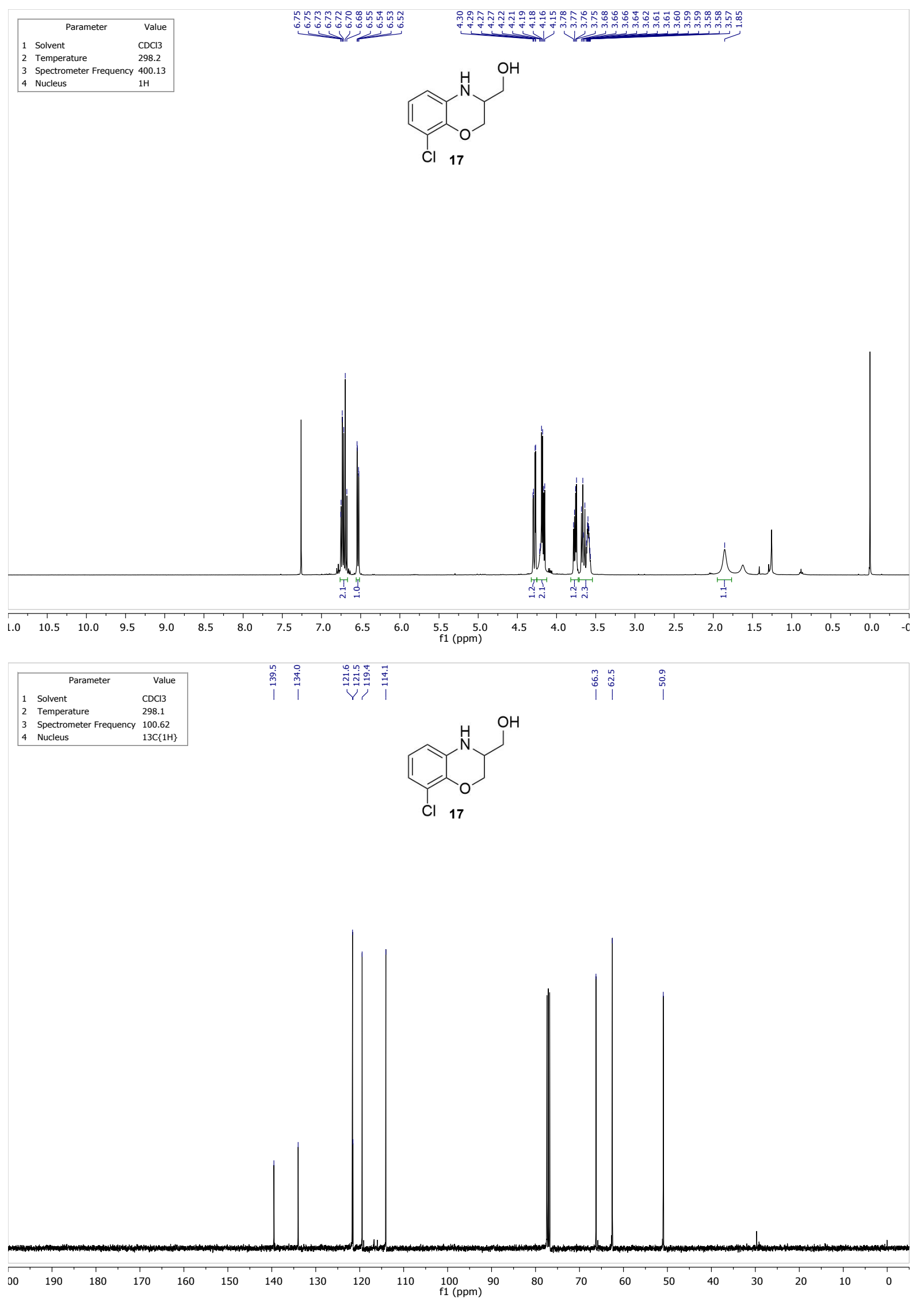


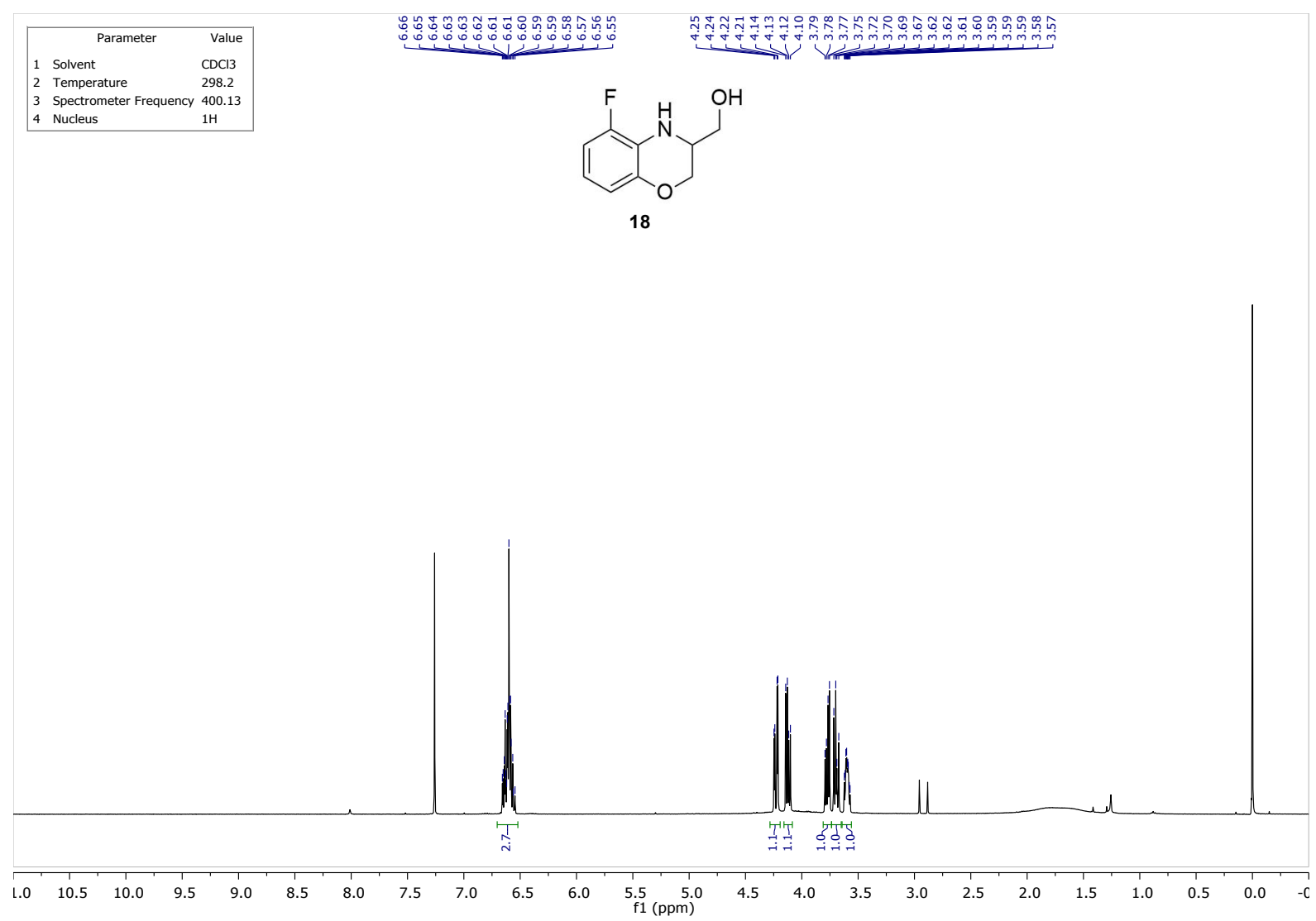

\begin{tabular}{|c|c|c|}
\hline & Parameter & Value \\
\hline 1 & Solvent & $\mathrm{CDCl3}$ \\
\hline 2 & Temperature & 298.2 \\
\hline 3 & Spectrometer Frequency & 376.53 \\
\hline 4 & Nucleus & $19 \mathrm{~F}$ \\
\hline
\end{tabular}

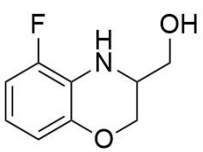

18

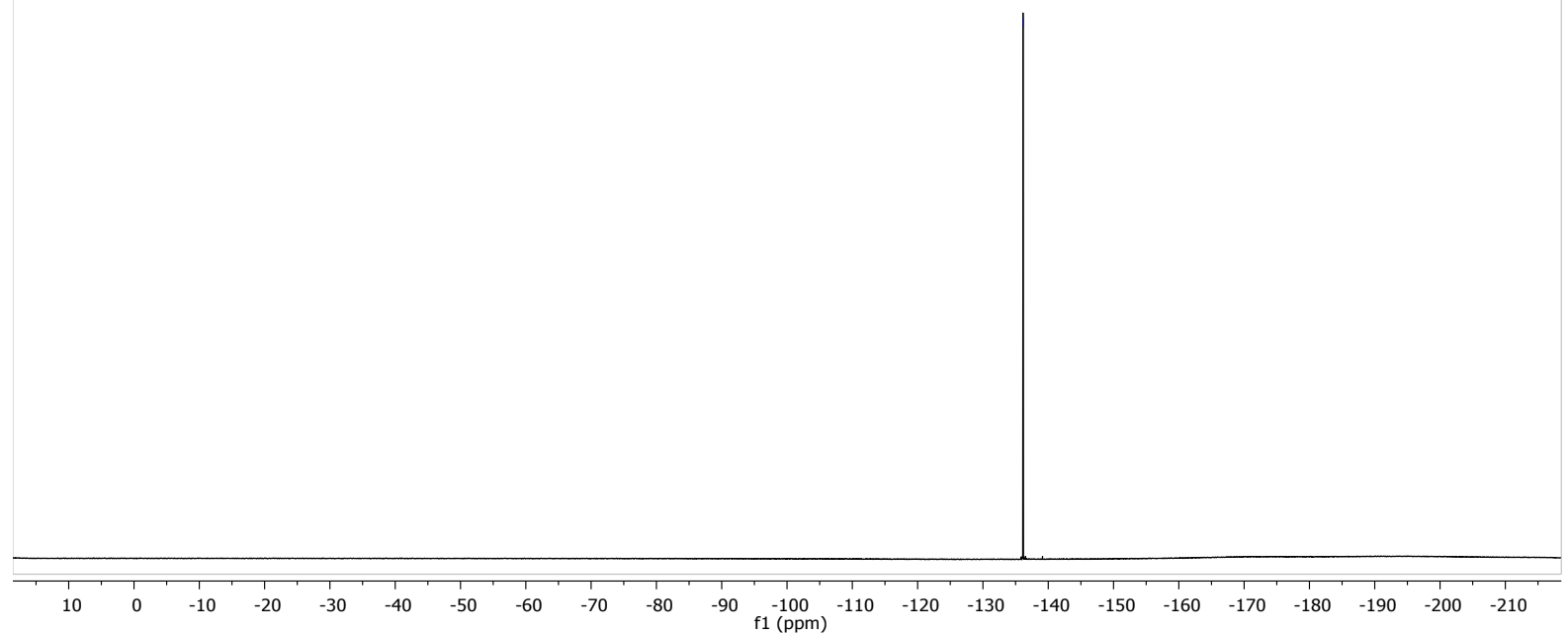




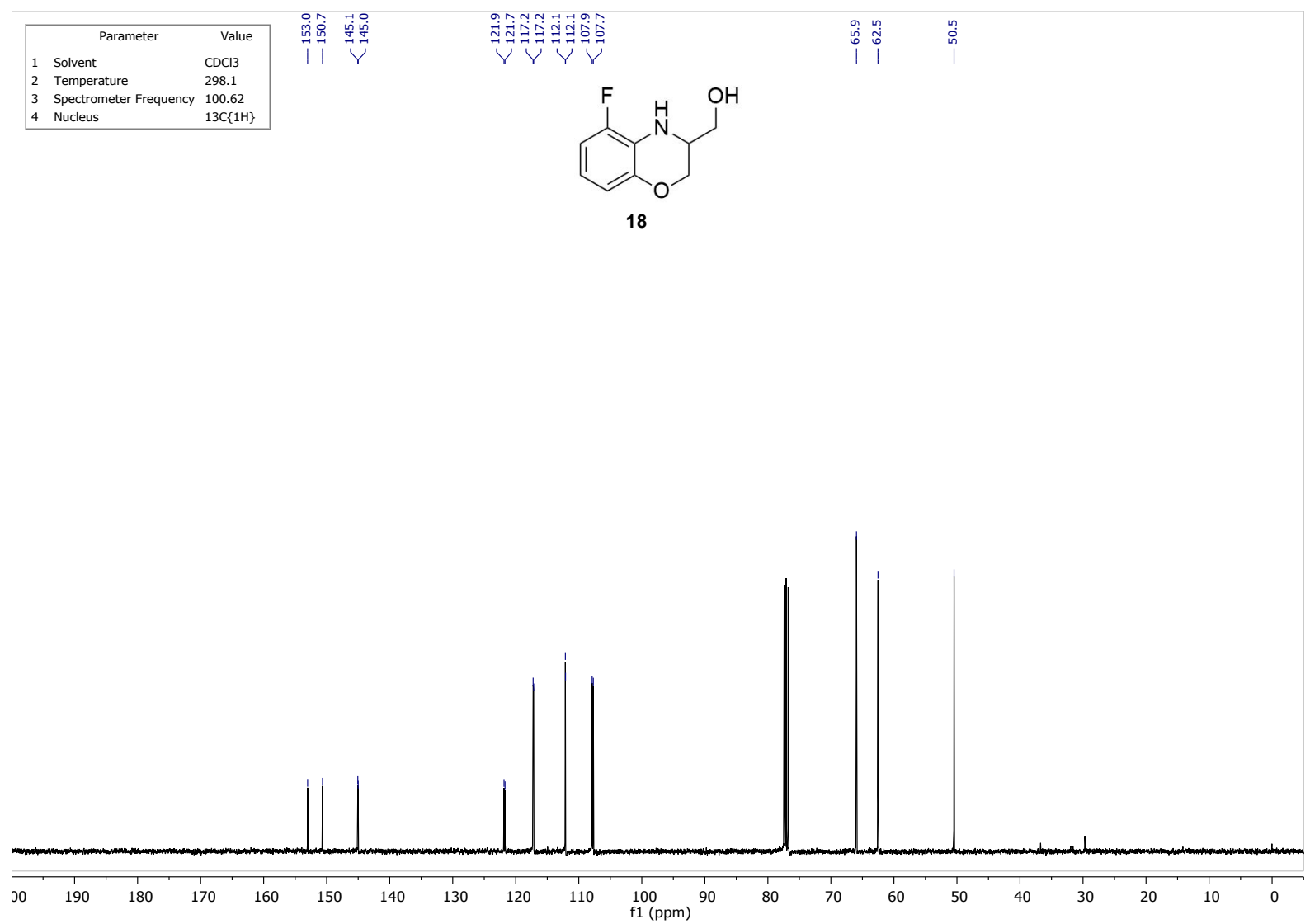




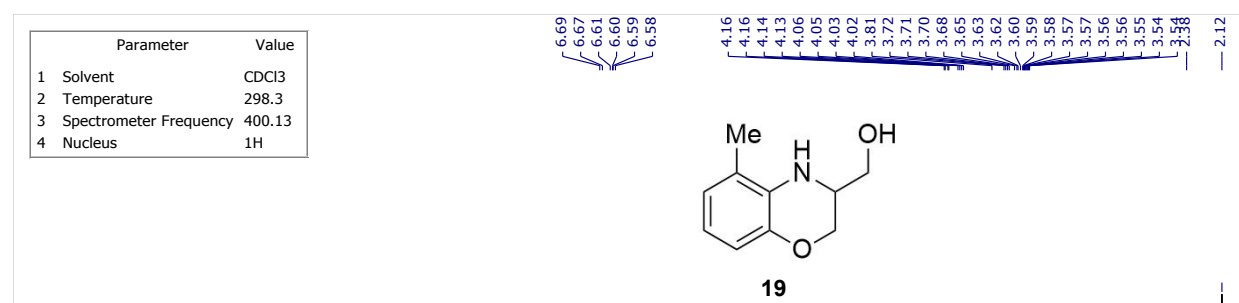

19
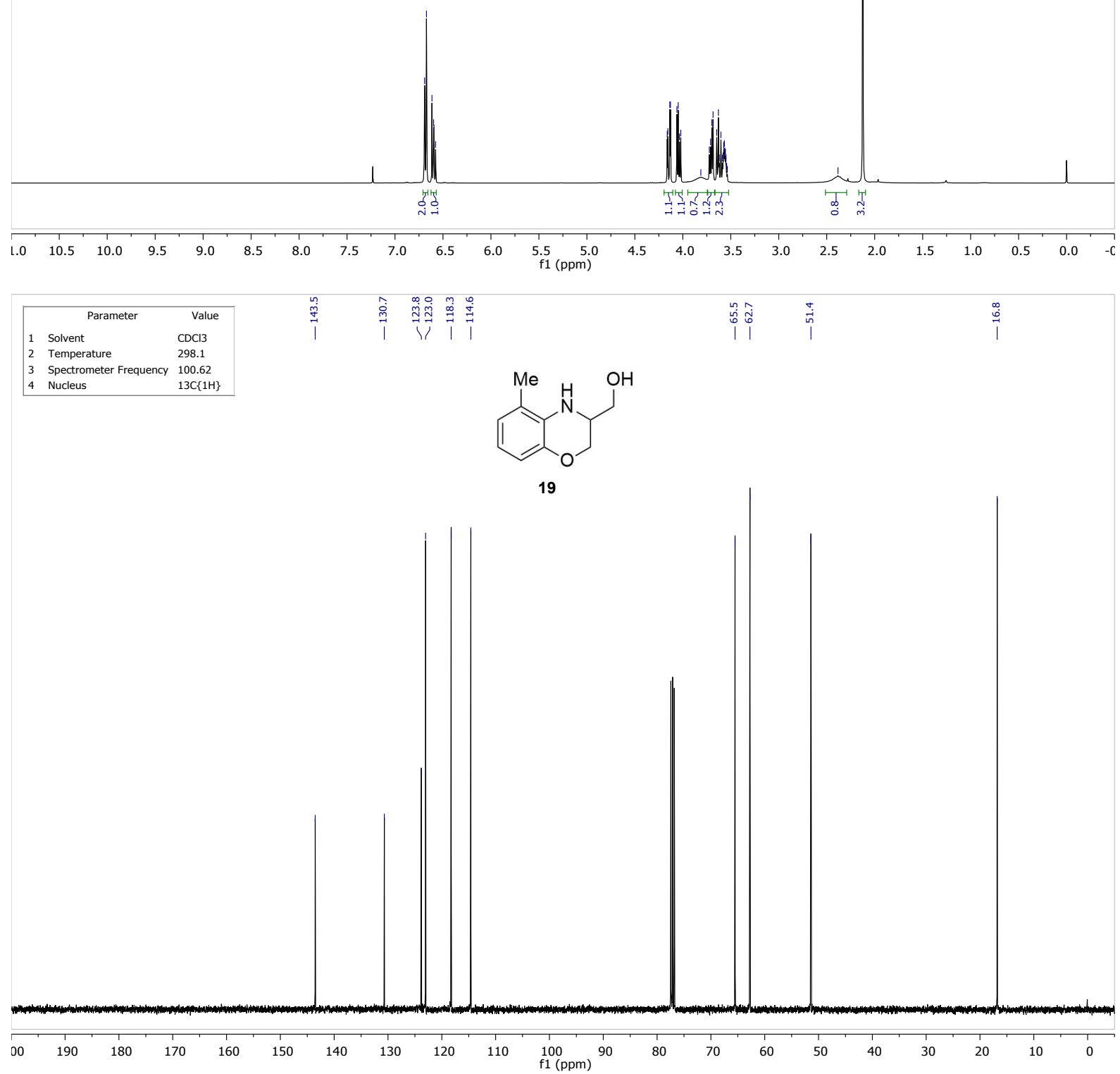

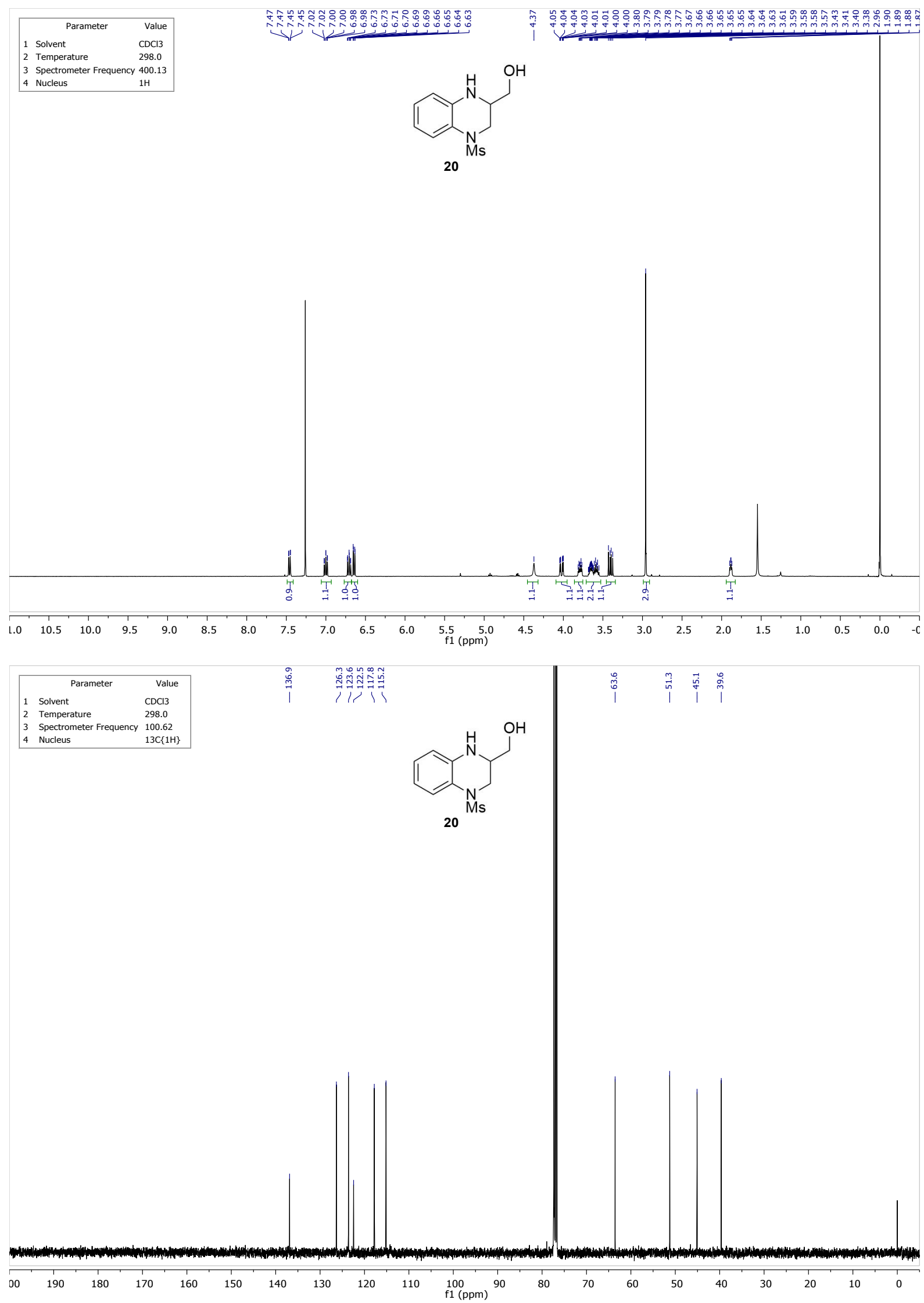

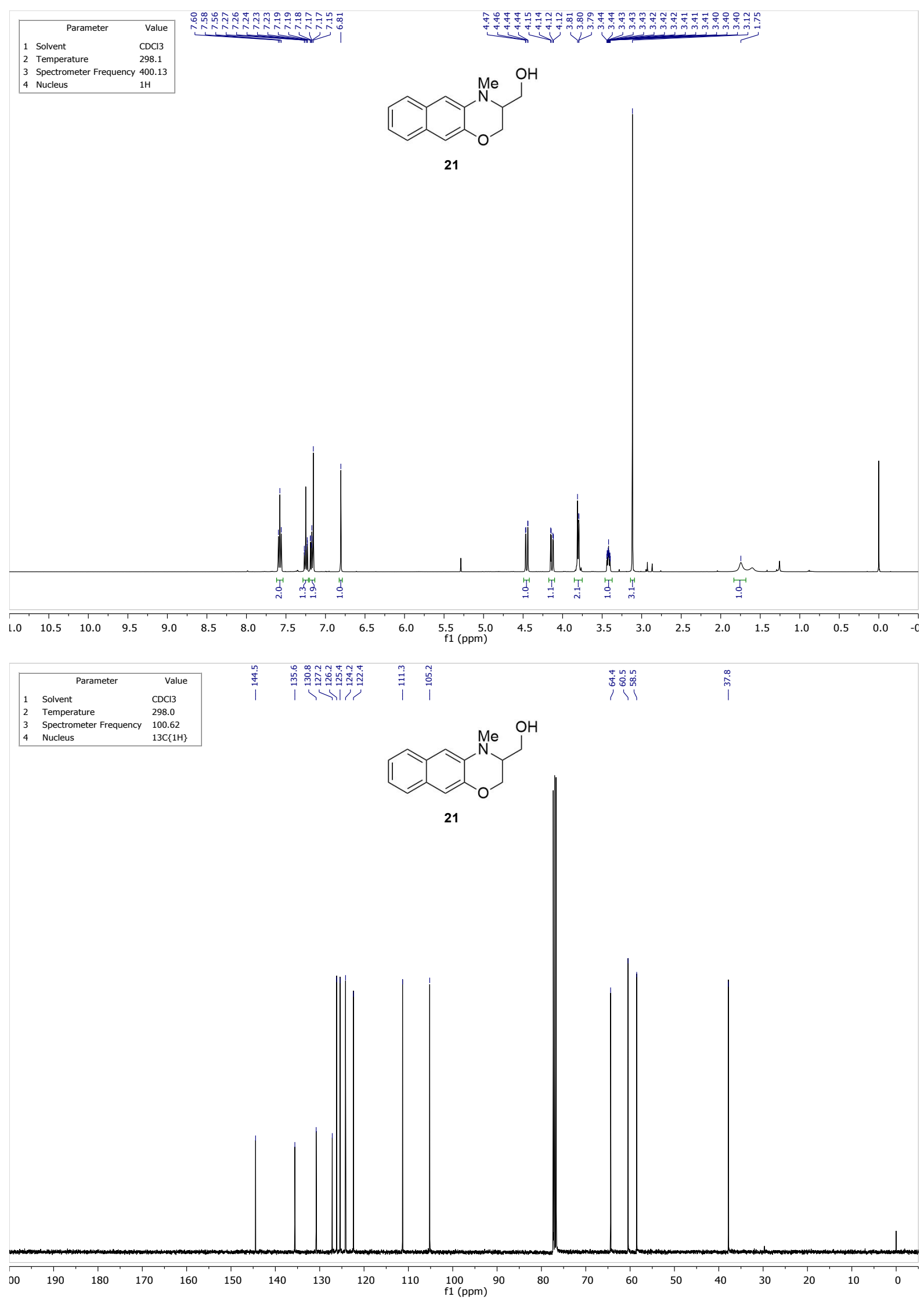

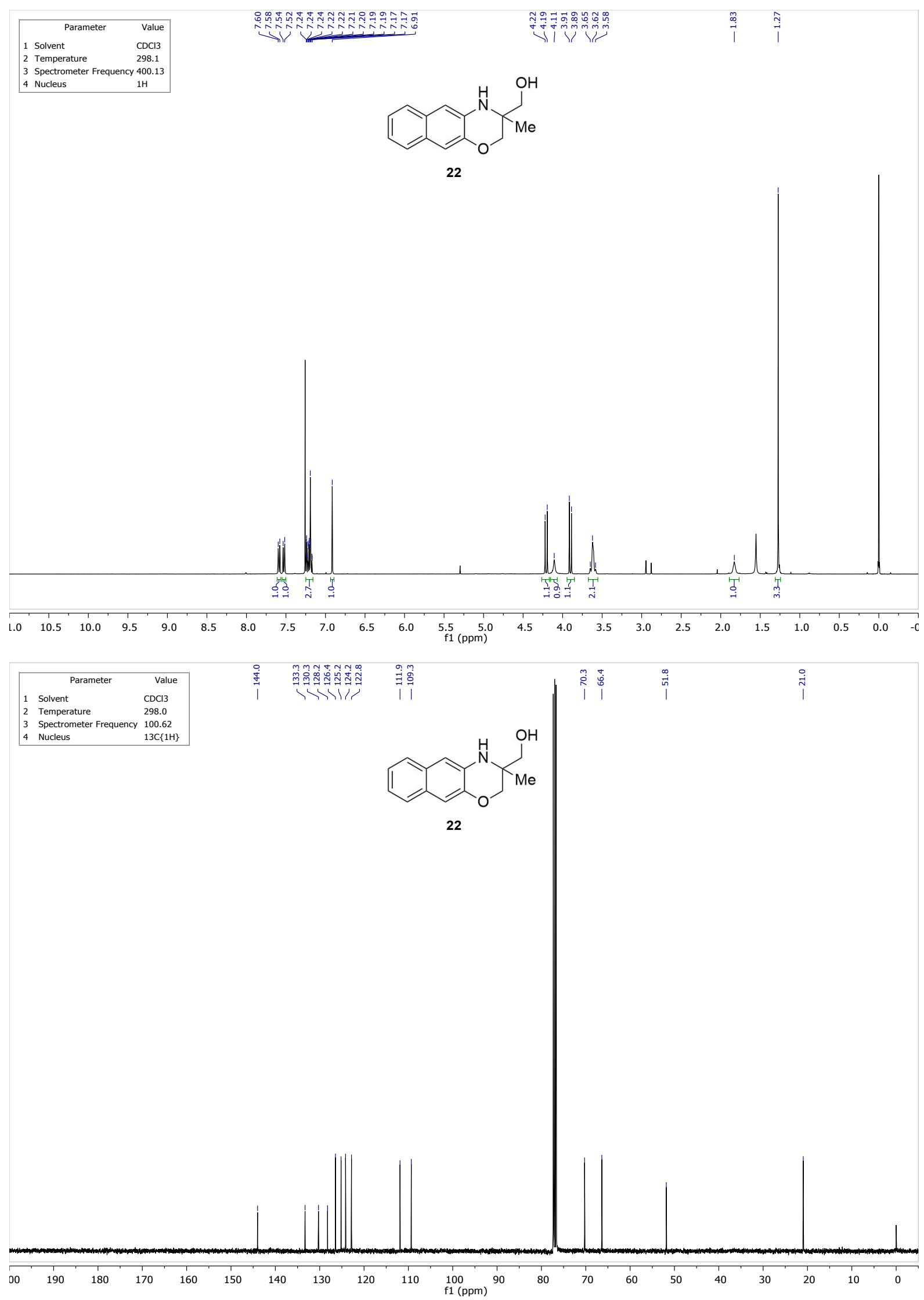

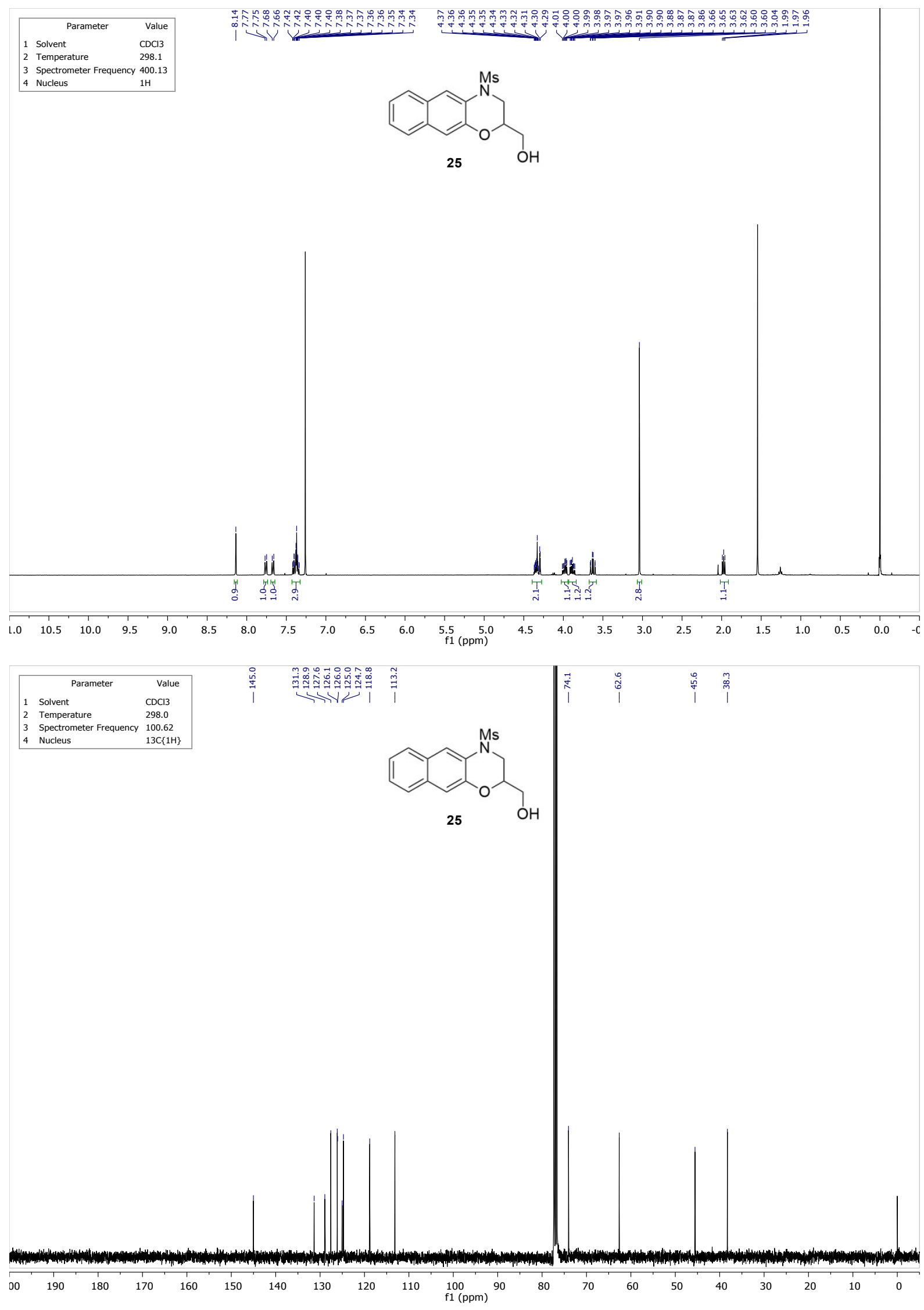

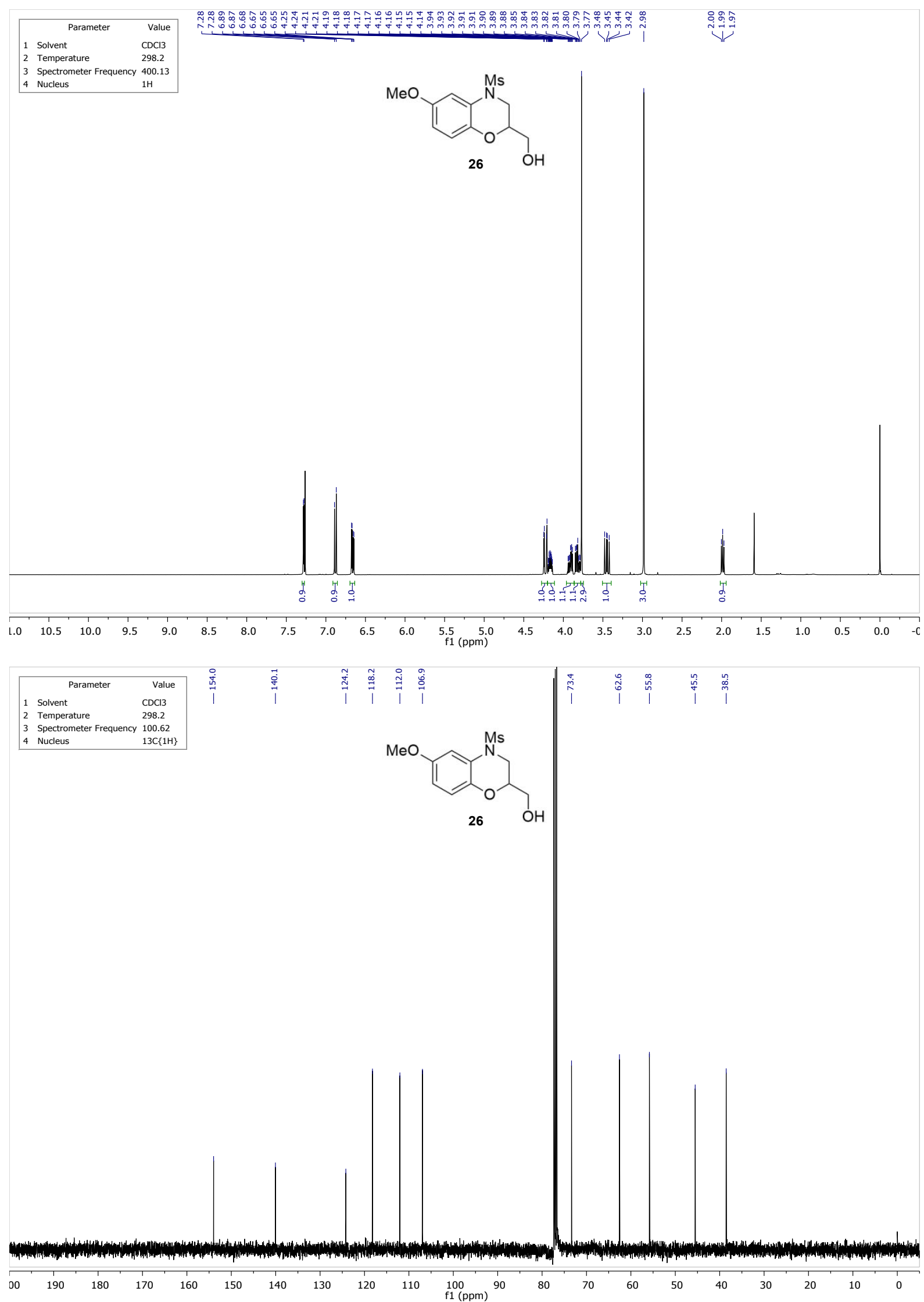

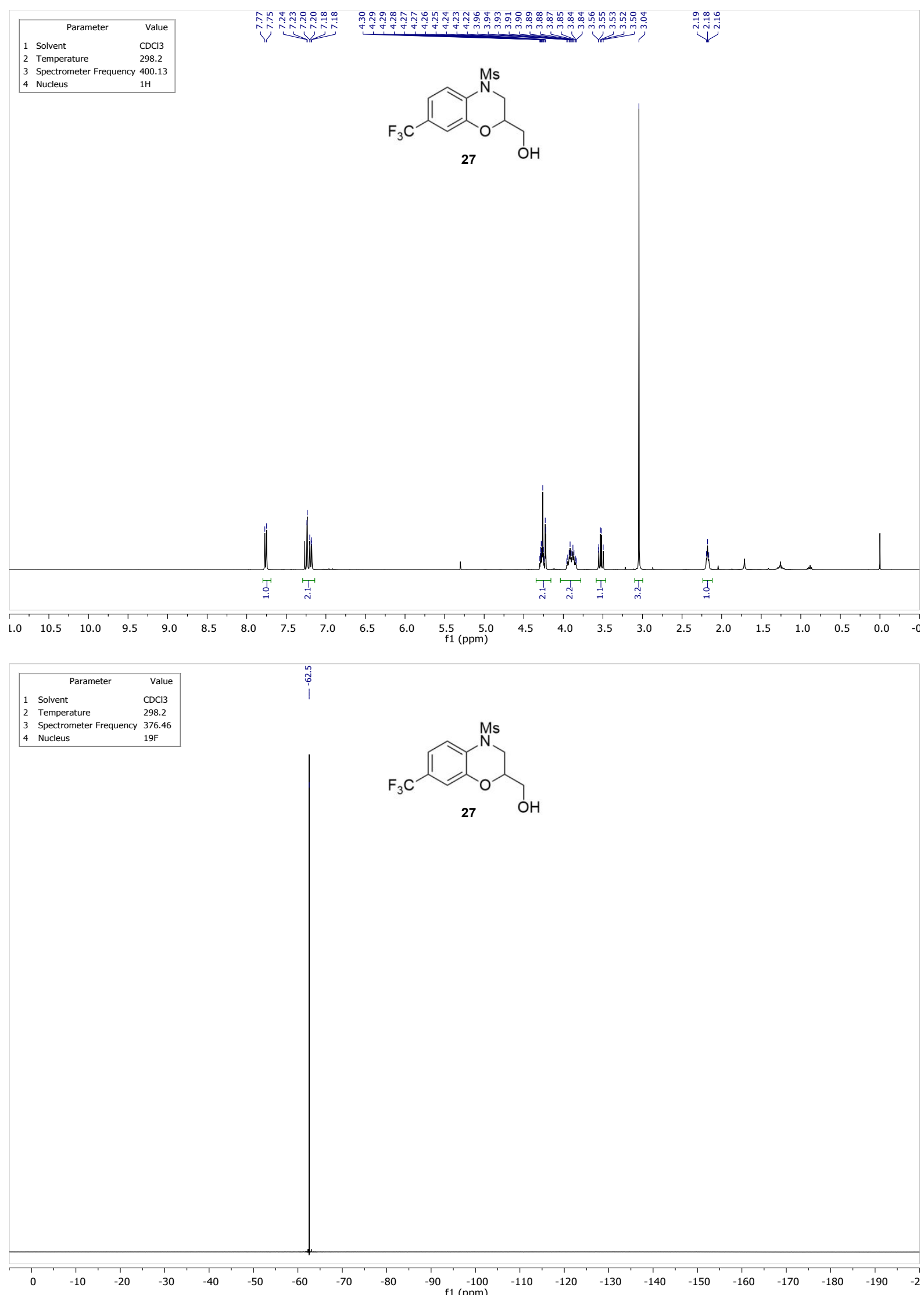


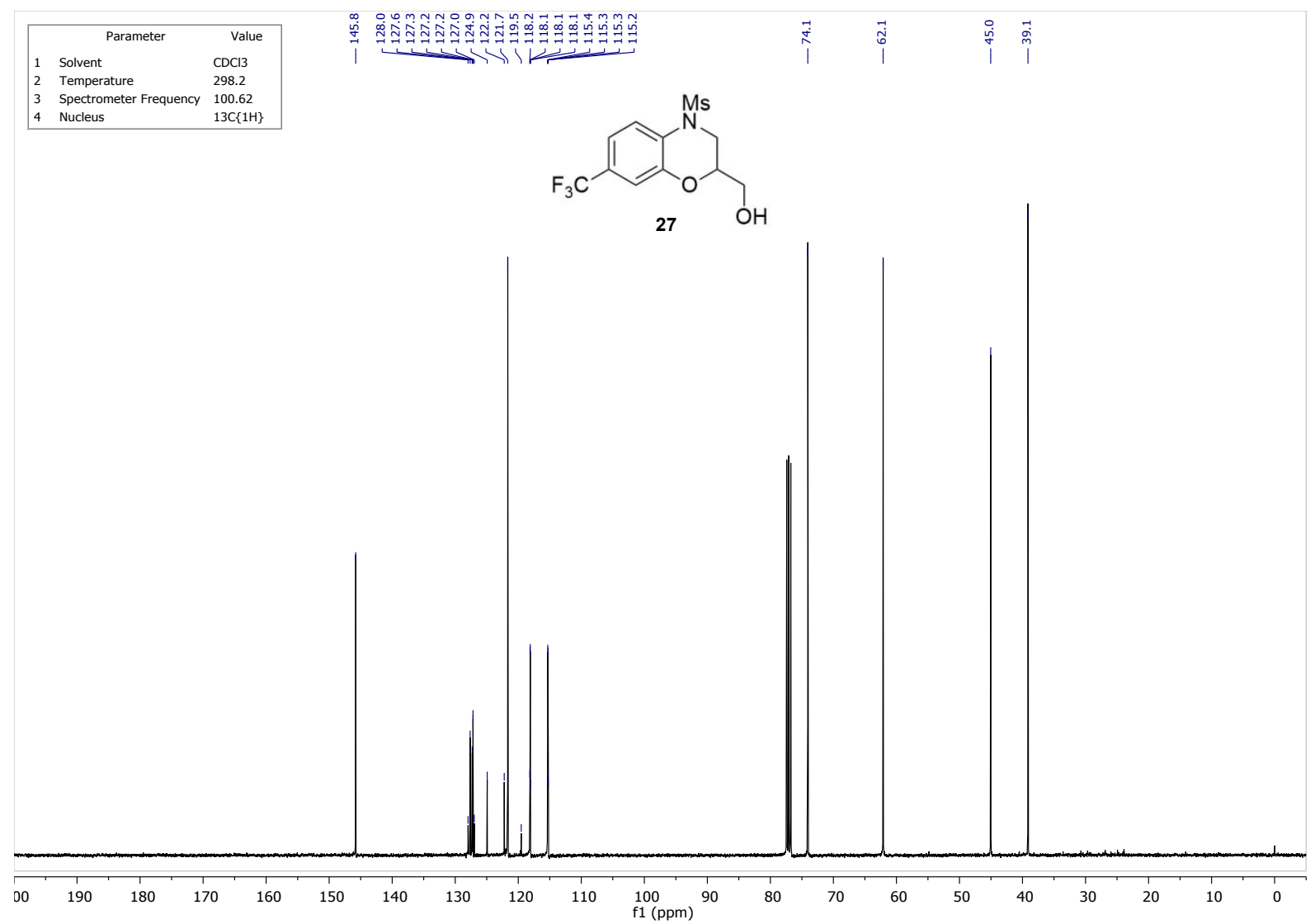




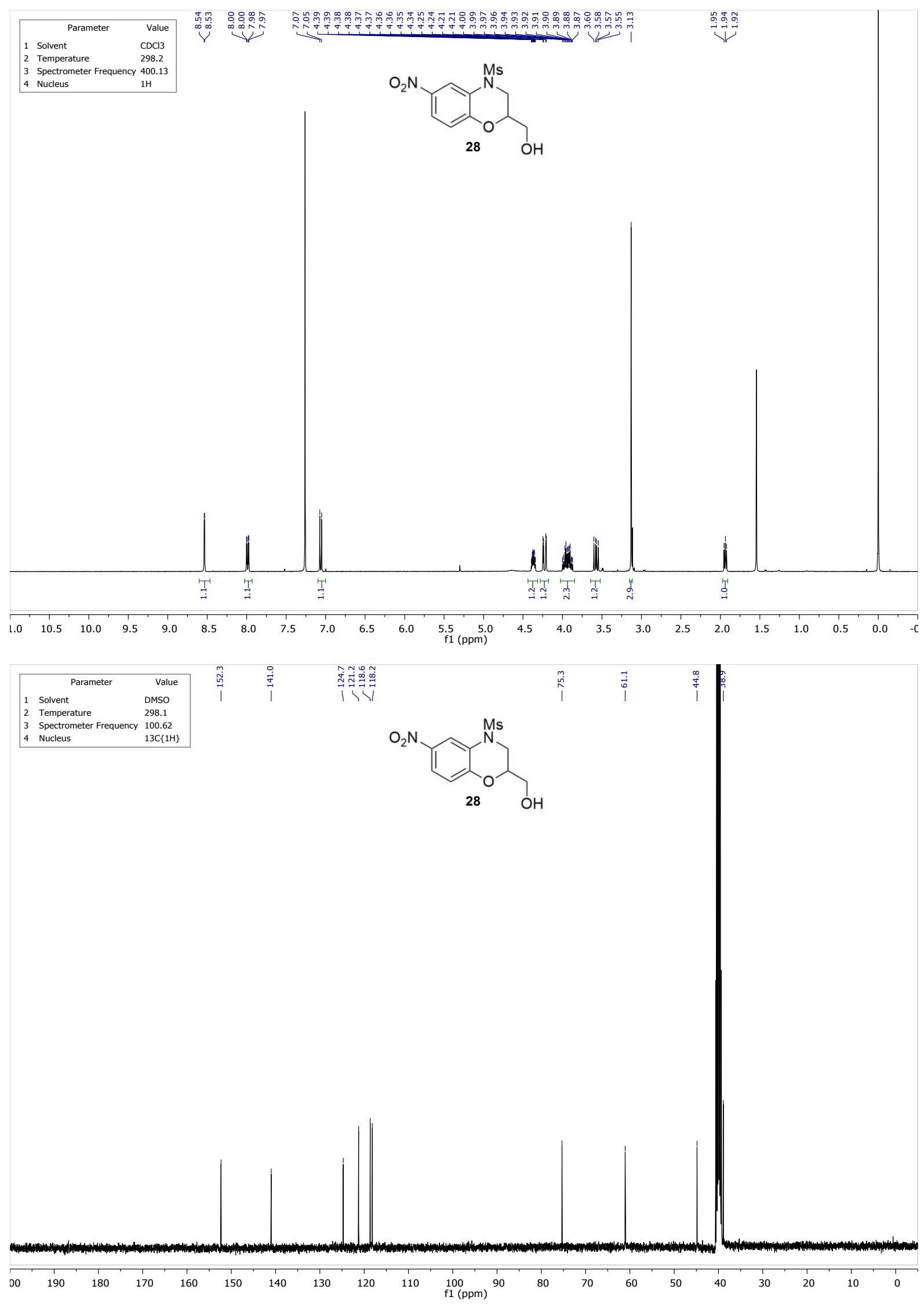



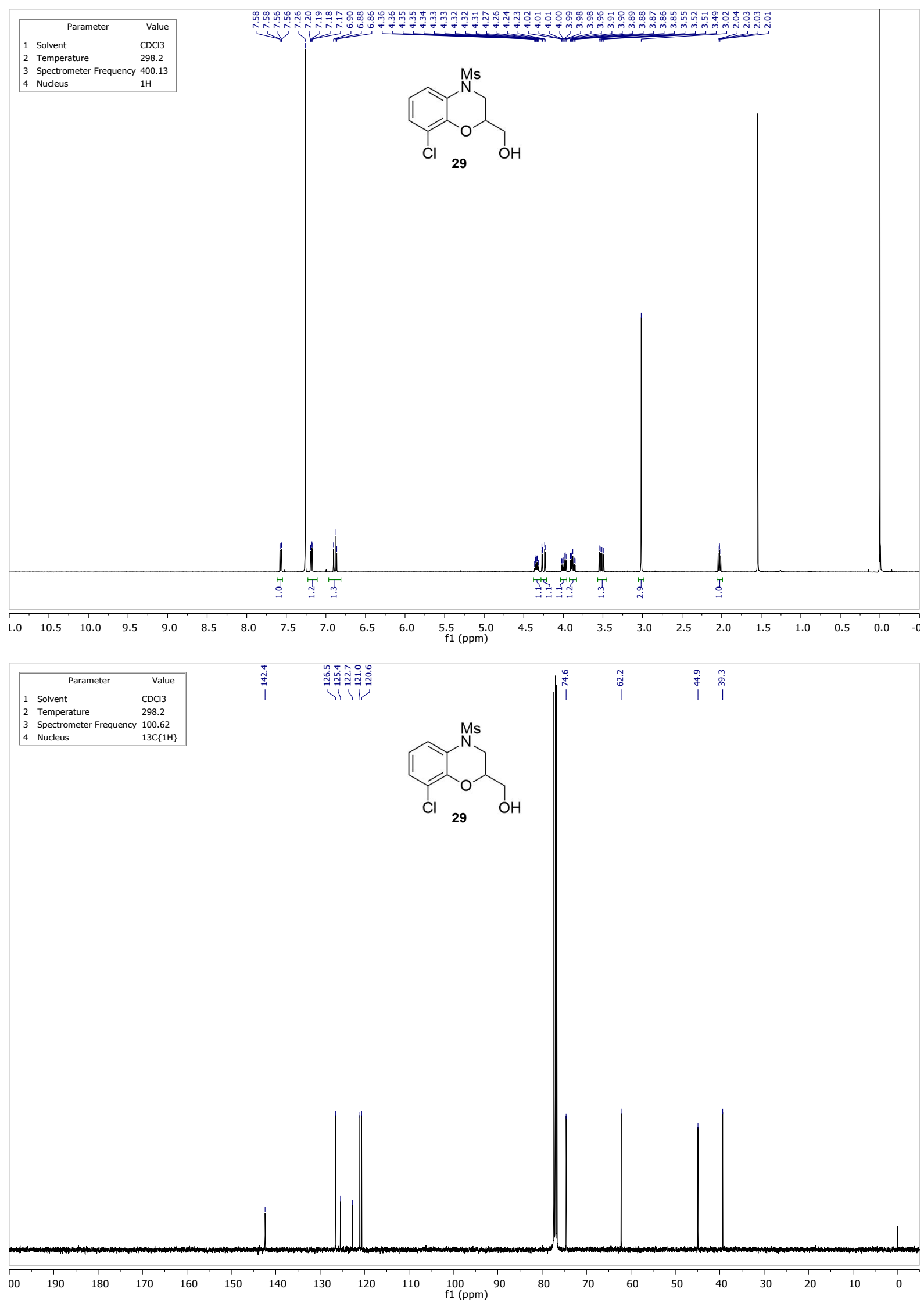

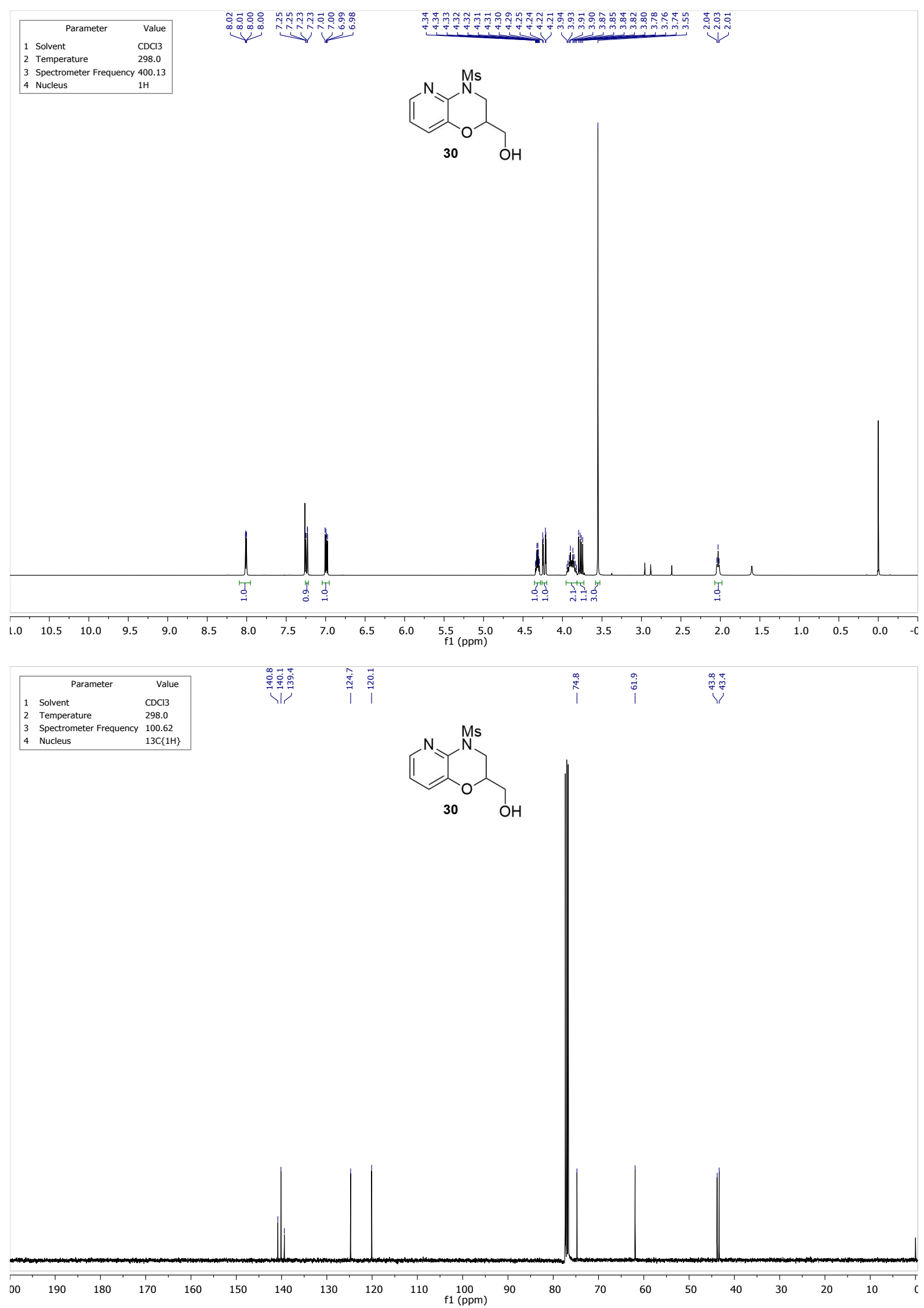

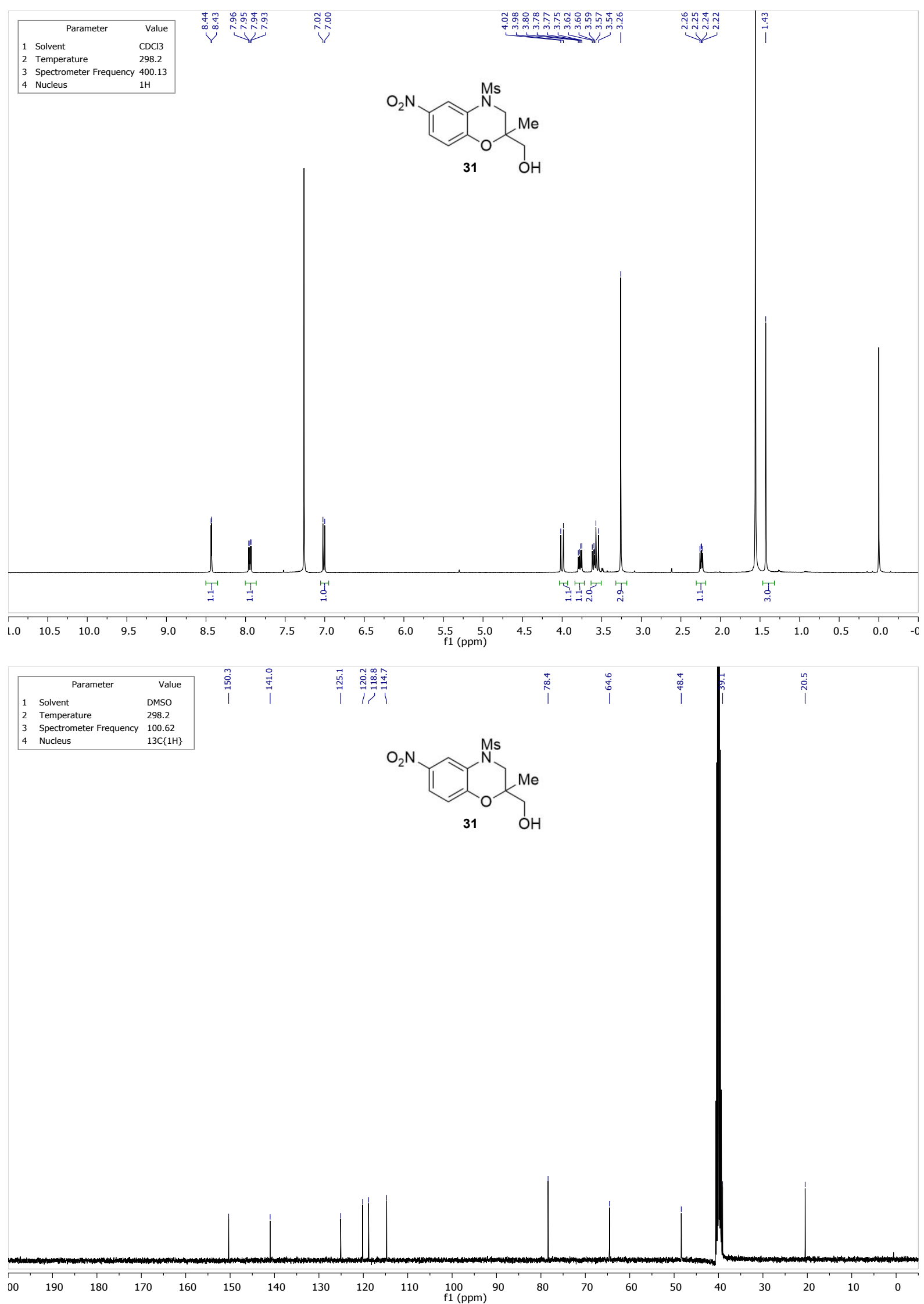

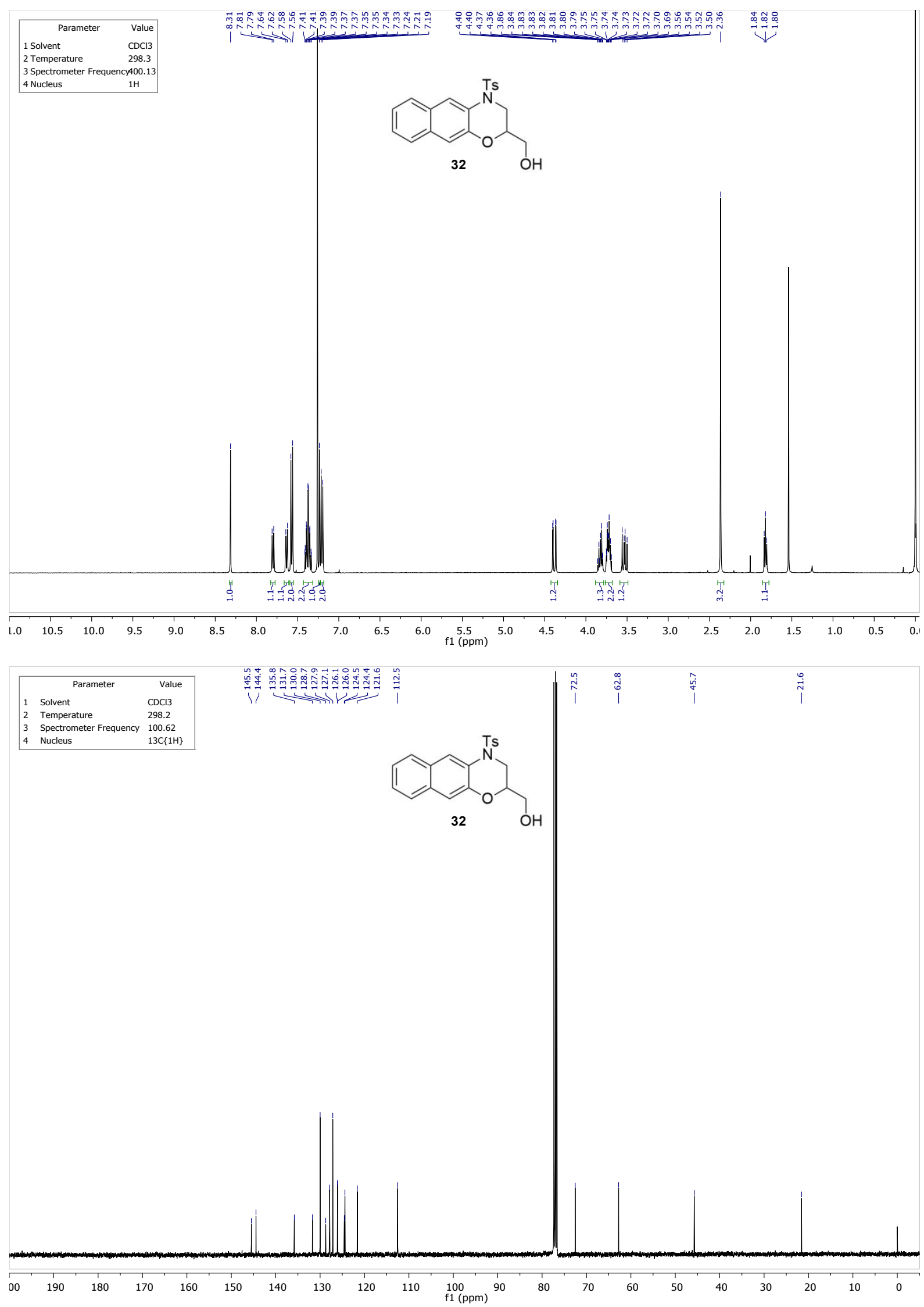

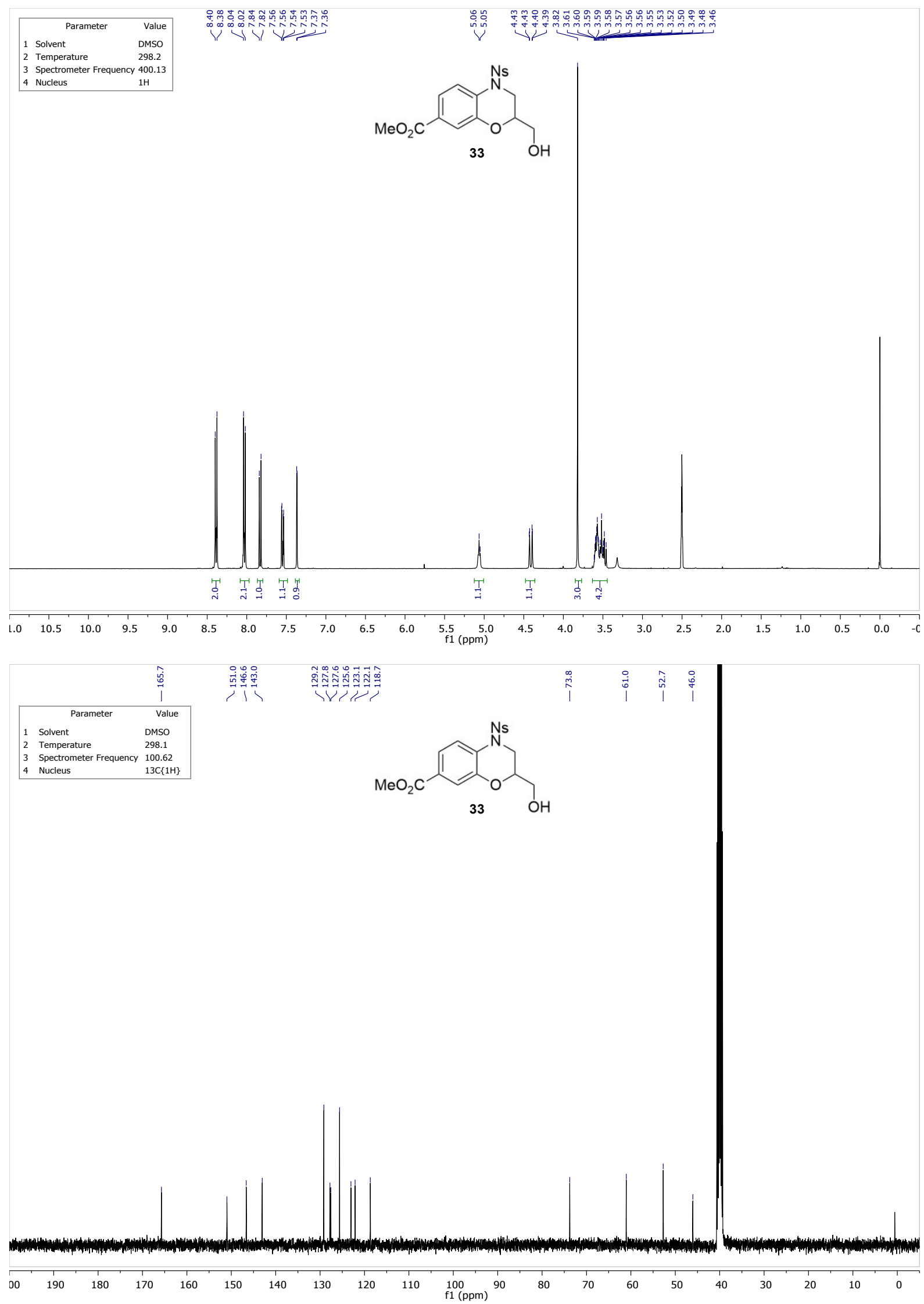
${ }^{1}$ Ramdas, V.; Loriya, R; Banerjee, M.; Chaure, G.; Das, A.; Joshi, A.; Walke, D.; Datrange, L.; Patil, P; Palle, V.; Kamboj, R. Indanyl Compounds as Voltage Gated Sodium Channel Modulators. WO2018/163077. Sep 13, 2018. 